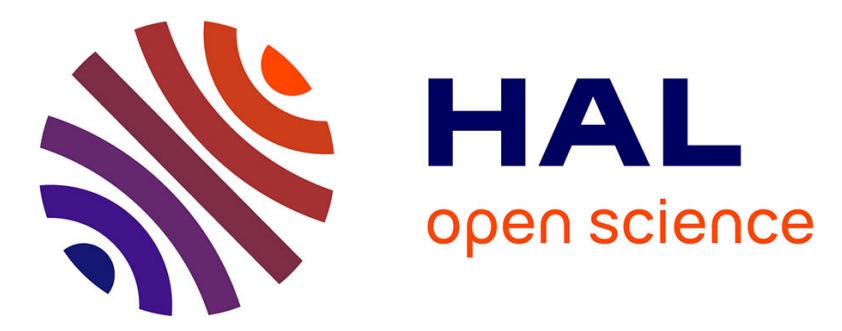

\title{
An energy-momentum conserving algorithm for non-linear hypoelastic constitutive models
}

Ludovic Noels, Laurent Stainier, Jean-Philippe Ponthot

\section{To cite this version:}

Ludovic Noels, Laurent Stainier, Jean-Philippe Ponthot. An energy-momentum conserving algorithm for non-linear hypoelastic constitutive models. International Journal for Numerical Methods in Engineering, 2004, 59 (1), pp.83-114. 10.1002/nme.869 . hal-01004990

\section{HAL Id: hal-01004990 \\ https://hal.science/hal-01004990}

Submitted on 5 Nov 2021

HAL is a multi-disciplinary open access archive for the deposit and dissemination of scientific research documents, whether they are published or not. The documents may come from teaching and research institutions in France or abroad, or from public or private research centers.
L'archive ouverte pluridisciplinaire HAL, est destinée au dépôt et à la diffusion de documents scientifiques de niveau recherche, publiés ou non, émanant des établissements d'enseignement et de recherche français ou étrangers, des laboratoires publics ou privés.

\section{다)(1) $(5$}

Distributed under a Creative Commons Attribution - NonCommercial| 4.0 International 


\title{
An energy-momentum conserving algorithm for non-linear hypoelastic constitutive models
}

\author{
L. Noels, L. Stainier and J. P. Ponthot*, \\ University of Liège, LTAS-Thermomechanics, Chemin des Chevreuils 1, B-4000 Liège, Belgium
}

\begin{abstract}
This paper presents an extension of the energy momentum conserving algorithm, usually developed for hyperelastic constitutive models, to the hypoelastic constitutive models. For such a material no potential can be defined, and thus the conservation of the energy is ensured only if the elastic work of the deformation can be restored by the scheme. We propose a new expression of internal forces at the element level which is shown to verify this property. We also demonstrate that the work of plastic deformation is positive and consistent with the material model. Finally several numerical applications are presented.
\end{abstract}

KEY WORDS: energy-momentum conserving; dynamics; hypoelastic constitutive model; large strain plasticity; finite elements

\section{INTRODUCTION}

One can resort to two families of algorithms to integrate the equations of evolution of dynamical systems: the implicit family and the explicit family. In this paper, we focus on the implicit family. The most widely used implicit algorithm is the Newmark algorithm [1] (see also for examples References [2-4]). Nevertheless, the total energy of a dynamical system, whose evolution equations are integrated by this algorithm, generally exhibits oscillations in time, even if the amplitude of these oscillations is limited for linear systems [5]. For non-linear models, Belytschko and Schoeberle in Reference [6] and Hughes in Reference [7] proved that the discrete energy is bounded if it remains positive. Nevertheless larger instabilities can arise, leading to divergence of the numerical simulation. Moreover, for a step between times $t_{n}$ and $t_{n+1}$, the angular momentum is conserved between the times $t_{n-(1 / 2)}$ and $t_{n+(1 / 2)}$ but not between the times of computation $t_{n}$ and $t_{n+1}$ [8]. To avoid divergence due to the numerical instabilities, numerical damping was introduced, leading to the generalized- $\alpha$ methods $[2-4,9,10]$. Another method is to set the Newmark parameters so as to dissipate energy [11]. But these techniques have the disadvantage to also damp the physical modes, leading to a lack of accuracy. Therefore a new kind of dynamics integration algorithm has appeared that

\footnotetext{
*Correspondence to: J. P. Ponthot, University of Liège, LTAS-Thermomechanics, Chemin des Chevreuils 1, B-4000 Liège, Belgium.

†E-mail: jp.ponthot@ulg.ac.be
} 
verifies the mechanical laws of conservation (i.e. conservation of linear momentum, angular momentum and total energy) and that remains stable in the non-linear range.

The first algorithm verifying these properties was described by Simo and Tarnow $[12,13]$. They called this algorithm energy momentum conserving algorithms or EMCA. It consists in a mid-point scheme with an adequate evaluation of the internal forces. This adequate evaluation was given for a Saint Venant-Kirchhoff hyperelastic material. This scheme was further extended to shells [14-17], to composite laminates [18] and to multi-body dynamics [10,19]. A generalization to other hyperelastic models was given by Laursen [20], who iteratively solves a new equation for each Gauss point to determine the adequate second Piola-Kirchhoff stress tensor. Another solution that avoids this iterative procedure leads to a general formulation of the second Piola-Kirchhoff stress tensor, as given by Gonzalez and Simo [21,22]. This formulation is valid for general hyperelastic materials. The EMCA was recently extended to dynamic finite deformation plasticity by Meng and Laursen [23]. In such a formulation, the algorithm remains energy conserving when no plastic deformation occurs, and dissipates energy in a manner consistent with the physical model in use (sic.) when plastic deformation occurs. The same method was applied to simulate non-frictional and frictional contact interactions by Armero and Petocz [24,25] and by Laursen and Chawla [26-28]. Numerical dissipation was also introduced in these conserving algorithms by Armero and Romero [29-32]. This algorithm preserves the angular momentum, contrarily to the generalized- $\alpha$ algorithms and is called energy dissipative Momentum Conserving algorithm or EDMC. This EDMC method was extended to beams by Ibrahimbegovic and Mamouri [33]. Another solution to verify all conservation equations is to use the generalized- $\alpha$ method or the EDMC algorithm, but to augment these algorithms with energy and momentum constraints [16,34-36]. This solution is called either constraint energy momentum algorithm (CEMA) in the first case or modified energy-momentum method (MEMM) in the second case. In such an augmented method, the dissipated energy of the high frequency modes is added to the energy of the low frequency mode. Finally the conservative properties can be ensured by using Petrov-Galerkin finite element method applied in the Hamiltonian way as developed by Betsch and Steinmann [37-39], or by using a Runge-Kutta method as developed by Bottasso et al. [40,41].

All the conserving methods described above were established for hyperelastic materials. To our knowledge, they were never extended to hypoelastic materials. This paper proposes a new expression of the internal forces, ensuring the conservation laws of the mechanics for a hypoelastic constitutive model. In Section 2 the methodology for evaluation of the stress tensor in hypoelastic materials and its spatial integration is recalled. In Section 3 the midpoint scheme is explained. The relations that have to be verified by the internal forces to remain consistent with the conservation laws are also exposed. In Section 4, we show how to compute the internal forces to verify these relations for a hypoelastic material using the final rotation scheme. Moreover, we prove that this adaptation remains consistent when plastic deformation occurs. Finally numerical examples illustrate the advantages and the disadvantages of the conserving algorithm (Section 5).

\section{THE HYPOELASTIC MATERIAL MODEL}

First, the notations used in this paper are detailed. Next, the method used for computation of the stress tensor in hypoelastic materials is explained. The plastic deformations are taken 
into account. Finally, the spatial integration of this stress tensor to obtain the internal forces is established in a finite element framework.

\subsection{Preliminaries}

Let the configuration $n$ be the configuration computed after $n$ time steps (i.e. at time $t_{n}$ ). Let $x^{n}$ be the deformation mapping (co-ordinates) in the configuration $n$, and let $x^{n \xi}$ be the co-ordinates of the position for the node $\xi(\xi \varepsilon[1, N]$ with $N$ the number of nodes of the element) in the configuration $n$. With $\varphi^{\xi}$ the shape function evaluated at node $\xi$, it comes (Einstein's notations are used)

$$
\begin{aligned}
& x=\varphi^{\xi} x^{\xi} \\
& \dot{x}=\varphi^{\xi} \dot{x}^{\xi} \\
& \ddot{x}=\varphi^{\xi} \ddot{x}^{\xi}
\end{aligned}
$$

The gradient of deformation (two point tensor) $\mathbf{F}$ between configurations $m$ and $n$ is indicated by $\mathbf{F}_{m}^{n}$. This tensor is defined by

$$
\mathbf{F}_{m}^{n}=\frac{\partial x^{n}}{\partial x^{m}}
$$

The tensor $\mathbf{f}$ represents $\mathbf{F}^{-1}$. When $m$ refers to the initial configuration, the gradient of deformation is written

$$
\mathbf{F}_{0}^{n}=\frac{\partial x^{n}}{\partial x^{0}}
$$

with

$$
\mathbf{F}_{0}^{n}=\mathbf{F}_{m}^{n} \mathbf{F}_{0}^{m}
$$

According to the theorem of polar decomposition, this gradient tensor can be decomposed into a rotation tensor $\mathbf{R}$ and a symmetric positive definite deformation tensor $\mathbf{U}$ ( $\mathbf{I}$ is the identity tensor)

$$
\begin{aligned}
\mathbf{F}_{m}^{n} & =\mathbf{R}_{m}^{n} \mathbf{U}_{m}^{n} \\
\mathbf{U}_{m}^{n} & =\mathbf{U}_{m}^{n \mathrm{~T}} \\
\mathbf{R}_{m}^{n \mathrm{~T}} \mathbf{R}_{m}^{n} & =\mathbf{I}
\end{aligned}
$$

The determinant of $\mathbf{F}_{m}^{n}$ is denoted by the scalar $J_{m}^{n}$. The relation between the density $\rho$ of the body and this determinant is

$$
\rho^{n}=\frac{\rho^{0}}{J_{0}^{n}}
$$


The Green-Lagrange strain tensor $\mathbf{G L}_{m}^{n}$ is defined as

$$
\begin{aligned}
\mathbf{G L}_{m}^{n} & =\frac{1}{2}\left[\mathbf{F}_{m}^{n}{ }^{\mathrm{T}} \mathbf{F}_{m}^{n}-\mathbf{I}\right] \\
& =\frac{1}{2}\left[\mathbf{U}_{m}^{n} \mathbf{U}_{m}^{n}-\mathbf{I}\right]
\end{aligned}
$$

and the Almansi strain tensor $\mathbf{A}_{m}^{n}$ is defined as

$$
\begin{aligned}
\mathbf{A}_{m}^{n} & =\frac{1}{2}\left[\mathbf{I}-\mathbf{f}_{m}^{n \mathrm{~T}} \mathbf{f}_{m}^{n}\right] \\
& =\mathbf{f}_{m}^{n \mathrm{~T}} \mathbf{G} \mathbf{L}_{m}^{n} \mathbf{f}_{m}^{n} \\
& =\frac{1}{2} \mathbf{R}_{m}^{n}\left[\mathbf{I}-\mathbf{U}_{m}^{n-1} \mathbf{U}_{m}^{n-1}\right] \mathbf{R}_{m}^{n \mathrm{~T}}
\end{aligned}
$$

The natural strain tensor $\mathbf{E}_{m}^{n}$ is also computed from $\mathbf{F}$ or might be computed from $\mathbf{G L}$ or from A

$$
\begin{aligned}
\mathbf{E}_{m}^{n} & =\frac{1}{2} \ln \left[\mathbf{F}_{m}^{n \mathrm{~T}} \mathbf{F}_{m}^{n}\right] \\
& =\frac{1}{2} \ln \left[2 \mathbf{G L}_{m}^{n}+\mathbf{I}\right] \\
& =-\frac{1}{2} \ln \left[\mathbf{I}-2 \mathbf{R}_{m}^{n \mathrm{~T}} \mathbf{A}_{m}^{n} \mathbf{R}_{m}^{n}\right]
\end{aligned}
$$

The Cauchy stress tensor is evaluated in the configuration $n$ and is referred to as $\boldsymbol{\Sigma}^{n}$. If the internal forces are pushed backward into the initial configuration, the stress tensor used is the second Piola-Kirchhoff tensor $(\mathbf{S})$, that is evaluated with respect to configuration $n$, as

$$
\mathbf{S}^{n}=J_{0}^{n} \mathbf{f}_{0}^{n} \boldsymbol{\Sigma}^{n} \mathbf{f}_{0}^{n \mathrm{~T}}
$$

\subsection{Stress tensor computation}

By definition, for a hyperelastic material, there exists a potential $\phi(\mathbf{G L})$ from which the second Piola-Kirchhoff stress tensor is computed

$$
\mathbf{S}^{n}=\rho^{0} \frac{\partial \phi\left(\mathbf{G L}_{0}^{n}\right)}{\partial \mathbf{G L}_{0}^{n}}
$$

For the hypoelastic constitutive laws, the Cauchy stress tensor is computed from a stress increment $\boldsymbol{\Delta} \boldsymbol{\Sigma}_{n}^{n+1}$ between two successive configurations. The final rotation scheme [11,42-45] is defined by the following relation:

$$
\boldsymbol{\Sigma}^{n+1}=\mathbf{R}_{n}^{n+1}\left[\boldsymbol{\Sigma}^{n}+\Delta \boldsymbol{\Sigma}_{n}^{n+1}\right] \mathbf{R}_{n}^{n+1^{\mathrm{T}}}
$$

This scheme presents some important properties [45]:

(i) it is incrementally objective (i.e. the stress tensor is exactly updated for a rigid body motion);

(ii) no parasitic volume variation is generated (i.e. the scheme does not lead to a variation of the volume for a rigid motion). 
If the material behaviour is elastic, the stress increment is deduced from the natural strain tensor

$$
\Delta \boldsymbol{\Sigma}_{n}^{n+1}=\mathscr{H}: \mathbf{E}_{n}^{n+1}
$$

with $\mathscr{H}$ the Hooke fourth order tensor ( $k$ is the bulk modulus and $g$ the shear modulus)

$$
\mathscr{H}_{i j k l}=k \delta_{i j} \delta_{k l}+g\left[\delta_{i k} \delta_{j l}+\delta_{i l} \delta_{j k}-\frac{2}{3} \delta_{i j} \delta_{k l}\right]
$$

and the operation $\mathscr{H}: \mathbf{E}$ defined by $\mathscr{H}_{i j k l} \mathbf{E}_{k l}$. For an elastoplastic or elastoviscoplastic material, relation (12) and relation (13) can only be directly used when the material remains elastic. If $J 2$ plastic deformations occur, relation (12) becomes

$$
\boldsymbol{\Sigma}^{n+1}=\mathbf{R}_{n}^{n+1}\left[\boldsymbol{\Sigma}^{n}+\Delta \boldsymbol{\Sigma}_{n}^{n+1}-\mathbf{s}^{\mathrm{c}}\right] \mathbf{R}_{n}^{n+1}{ }^{\mathrm{T}}
$$

where $\mathbf{s}^{\mathrm{c}}$ is the purely deviatoric correction tensor resulting from the radial return mapping [45-48]. It is evaluated by the following method: the elastic predictor $\mathbf{s}^{\mathrm{e}}$ is defined by the deviatoric part of $\boldsymbol{\Sigma}^{n}+\boldsymbol{\Delta} \boldsymbol{\Sigma}_{n}^{n+1}$ where $\boldsymbol{\Delta} \boldsymbol{\Sigma}_{n}^{n+1}$ is given by (13). If $\boldsymbol{\alpha}$ is the heredity back stress tensor, the normal tensor $\mathbf{N}$ is defined by

$$
\mathbf{N}=\frac{\mathbf{s}^{\mathrm{e}}-\boldsymbol{\alpha}}{\sqrt{\left[\mathbf{s}^{\mathrm{e}}-\alpha\right]:\left[\mathbf{s}^{\mathrm{e}}-\alpha\right]}}
$$

where the operation $\mathbf{a}: \mathbf{b}$ is defined as $\mathbf{a}_{i j} \mathbf{b}_{i j}$. If the scalar $\varepsilon^{\mathrm{p}}$ is the equivalent plastic strain, if the scalar $\Sigma_{v}$, function of $\varepsilon^{\mathrm{p}}$, is the subsequent von Mises yield stress, and if $\bar{\alpha}$, function of $\varepsilon^{\mathrm{p}}$, is the equivalent heredity, then the scalar $\gamma$ can be defined such as to have $[11,45]$

$$
\begin{aligned}
\varepsilon^{\mathrm{p}^{n+1}} & =\varepsilon^{\mathrm{p} n}+\sqrt{\frac{2}{3} \gamma} \\
\sum_{v}^{n+1}\left(\varepsilon^{\mathrm{p}^{n+1}}\right) & =\Sigma_{v}^{n+1}(\gamma) \\
\boldsymbol{\alpha}^{n+1} & =\boldsymbol{\alpha}^{n}+\sqrt{\frac{2}{3}}\left[\bar{\alpha}\left(\varepsilon^{\mathrm{p} n+1}\right)-\bar{\alpha}\left(\varepsilon^{\mathrm{p}^{n}}\right)\right] \mathbf{N} \\
\mathbf{s}^{\mathbf{c}} & =2 g \gamma \mathbf{N}
\end{aligned}
$$

where the scalar value of $\gamma$ is solved from the von Mises criterion [45] evaluated at time $t_{n+1}$

$$
\left[\mathbf{s}^{\mathrm{e}}-2 g \gamma \mathbf{N}-\boldsymbol{\alpha}^{n+1}(\gamma)\right]:\left[\mathbf{s}^{\mathrm{e}}-2 g \gamma \mathbf{N}-\boldsymbol{\alpha}^{n+1}(\gamma)\right]=\frac{2}{3}\left[\sum_{v}^{n+1}(\gamma)\right]^{2}
$$

Now we will establish the expression of the internal forces from the Cauchy stress tensor.

\subsection{Internal forces formulation}

Let $\delta u$ be an admissible virtual displacement, let $\delta W_{\mathrm{ext}}^{n}, \delta W_{\text {int }}^{n}$ and $\delta K^{n}$, respectively, be the virtual work of the external forces, the virtual work of the internal forces and the virtual work of inertia forces in the configuration $n$, let $b^{n}$ be the volumic forces, let $t^{n}$ be the surfacic tractions, let $V_{n}$ be the volume of the element and let $S_{n}$ be the boundary of the element. Then, the principle of the virtual work can be rewritten as [11]

$$
\delta K^{n}+\delta W_{\mathrm{int}}^{n}=\delta W_{\mathrm{ext}}^{n}
$$


with

$$
\begin{aligned}
\delta K^{n} & =\int_{V_{n}}\left\{\rho^{n} \ddot{x}^{n} * \delta u\right\} \mathrm{d} V_{n} \\
\delta W_{\text {ext }}^{n} & =\int_{V_{n}}\left\{\rho^{n} b^{n} * \delta u\right\} \mathrm{d} V_{n}+\int_{S_{n}}\left\{t^{n} * \delta u\right\} \mathrm{d} S_{n} \\
\delta W_{\text {int }}^{n} & =\int_{V_{n}}\left\{\Sigma^{n \mathrm{~T}}: \frac{\partial \delta u}{\partial x^{n}}\right\} \mathrm{d} V_{n}
\end{aligned}
$$

where the operation $a * b$ is defined by $a_{i} b_{i}$. Using (1), (6), the mass conservation law (i.e. $\left.\rho^{n} \mathrm{~d} V_{n}=\rho^{0} \mathrm{~d} V_{0}\right)$ and the spatial discretization of the virtual displacement (i.e. $\delta u=\varphi^{\xi} \delta u^{\xi}$ ), the virtual work of the inertia forces can be rewritten as

$$
\begin{aligned}
\delta K^{n} & =\int_{V_{0}}\left\{\rho^{0} \varphi^{\xi} \varphi^{\mu}\right\} \mathrm{d} V_{0}\left[\ddot{x}^{n}\right]^{\mu} * \delta u^{\xi} \\
& =M^{\xi \mu}\left[\ddot{x}^{n}\right]^{\mu} * \delta u^{\xi}
\end{aligned}
$$

where $M^{\xi \mu}$ is the mass matrix component relative at the nodes $\xi$ and $\mu$. The virtual work of external forces is expressed as

$$
\delta W_{\mathrm{ext}}^{n}=\left[F_{\mathrm{ext}}^{n}\right]^{\xi} * \delta u^{\xi}
$$

Finally, the internal forces variation can be rewritten as

$$
\begin{aligned}
\delta W_{\text {int }}^{n} & =\int_{V_{0}}\left\{\boldsymbol{\Sigma}^{n \mathrm{~T}}\left[\frac{\partial \varphi^{\xi}}{\partial x^{n}}\right]^{\mathrm{T}} J_{0}^{n}\right\} \mathrm{d} V_{0} * \delta u^{\xi} \\
& =\int_{V_{0}}\left\{\boldsymbol{\Sigma}^{n \mathrm{~T}} \mathbf{f}_{0}^{n \mathrm{~T}} D^{\xi} J_{0}^{n}\right\} \mathrm{d} V_{0} * \delta u^{\xi}
\end{aligned}
$$

where $D$ is the derivative of the shape function (in the reference configuration, i.e. $D^{\xi}=$ $\left.\partial \varphi^{\xi} / \partial x^{0}\right)$. Using relation (21)-(23), the balanced equation at node $\xi$ for the configuration $n$ leads to

$$
M^{\xi \mu}\left[\ddot{x}^{n}\right]^{\mu}=\left[F_{\text {ext }}^{n}-F_{\text {int }}^{n}\right]^{\xi}
$$

with the expression of internal forces given by

$$
\left[F_{\text {int }}^{n}\right]^{\xi}=\int_{V_{0}}\left\{\boldsymbol{\Sigma}^{n \mathrm{~T}} \mathbf{f}_{0}^{n \mathrm{~T}} D^{\xi} J_{0}^{n}\right\} \mathrm{d} V_{0}
$$

These expressions are valid for any time $t_{n}$.

\section{THE ENERGY-MOMENTUM CONSERVING SCHEME}

Equation (24) has to be resolved for successive time steps, but this integration should verify the conservation laws. First the mid-point scheme presented in Reference [12] is briefly 
recalled. Next the relations that the internal forces should verify to be consistent with the conservation laws are detailed.

\subsection{The mid-point scheme}

For an integration from time $t_{n}$ to time $t_{n}+\Delta t=t_{n+1}$, the relations between positions, velocities and accelerations are given by

$$
\begin{aligned}
x^{n+(1 / 2)} & =\frac{x^{n+1}+x^{n}}{2} \\
\dot{x}^{n+(1 / 2)} & =\frac{x^{n+1}-x^{n}}{\Delta t} \\
& =\frac{\dot{x}^{n+1}+\dot{x}^{n}}{2} \\
\ddot{x}^{n+(1 / 2)} & =\frac{\dot{x}^{n+1}-\dot{x}^{n}}{\Delta t} \\
& =\frac{\ddot{x}^{n+1}+\ddot{x}^{n}}{2}
\end{aligned}
$$

The balance law (24) for node $\xi$ is rewritten as

$$
M^{\xi \mu}\left[\ddot{x}^{n+(1 / 2)}\right]^{\mu}=\left[F_{\mathrm{ext}}^{n+(1 / 2)}-F_{\mathrm{int}}^{n+(1 / 2)}\right]^{\xi}
$$

Let $F^{n+(1 / 2)}\left(x^{n}, x^{n+1}\right)$ be the expression of the forces in configuration $n+\frac{1}{2}$. This expression depends both on the positions in configurations $n$ (i.e. $x^{n}$ ) and $n+1$ (i.e. $x^{n+1}$ ). The goal of the following section is to evaluate it for hypoelastic models. Systems (26) and (27) can be resolved by a predictor-corrector algorithms. The predicted values are

$$
\begin{aligned}
& x^{n+1}=x^{n}+\Delta t \dot{x}^{n}+\frac{\Delta t^{2}}{4} \ddot{x}^{n} \\
& \dot{x}^{n+1}=\dot{x}^{n}+\frac{\Delta t}{2} \ddot{x}^{n} \\
& \ddot{x}^{n+1}=0
\end{aligned}
$$

Residual for configuration $n+1$ is expressed as

$$
\Delta F^{\xi}=\frac{1}{2} M^{\xi \mu}\left[\ddot{x}^{n+1}+\ddot{x}^{n}\right]^{\mu}+\left[F_{\mathrm{int}}^{n+(1 / 2)}-F_{\mathrm{ext}}^{n+(1 / 2)}\right]^{\xi}
$$


Then, the corrections for the values at configuration $n+1$ are iteratively evaluated as

$$
\begin{aligned}
{\left[\mathbf{K}^{\xi \mu}+\frac{2}{\Delta t^{2}} M^{\xi \mu} \mathbf{I}\right] \Delta x^{\mu} } & =-\Delta F^{\xi} \\
{\left[x^{n+1}\right]^{\mu} } & \leftarrow\left[x^{n+1}+\Delta x\right]^{\mu} \\
{\left[\dot{x}^{n+1}\right]^{\mu} } & \leftarrow\left[\dot{x}^{n+1}+\frac{2}{\Delta t} \Delta x\right]^{\mu} \\
{\left[\ddot{x}^{n+1}\right]^{\mu} } & \leftarrow\left[\ddot{x}^{n+1}+\frac{4}{\Delta t^{2}} \Delta x\right]^{\mu}
\end{aligned}
$$

where $\mathbf{K}^{\xi \mu}$ is tangent stiffness matrix

$$
\mathbf{K}^{\xi \mu}=\frac{\partial\left[F_{\mathrm{int}}^{n+(1 / 2)}\right]^{\xi}}{\partial\left[x^{n+1}\right]^{\mu}}-\frac{\partial\left[F_{\mathrm{ext}}^{n+(1 / 2)}\right]^{\xi}}{\partial\left[x^{n+1}\right]^{\mu}}
$$

Equations (30) are solved iteratively until convergence of the iterations occurs, i.e. until

$$
\frac{\Delta F^{\xi} * \Delta F^{\xi}}{\left[F_{\mathrm{int}}^{n+(1 / 2)}\left(x^{n+1}\right)\right]^{\xi} *\left[F_{\mathrm{int}}^{n+(1 / 2)}\left(x^{n+1}\right)\right]^{\xi}+\left[F_{\mathrm{ext}}^{n+(1 / 2)}\left(x^{n+1}\right)\right]^{\xi} *\left[F_{\mathrm{ext}}^{n+(1 / 2)}\left(x^{n+1}\right)\right]^{\xi}}<\mathrm{Tol}
$$

where Tol is a user defined tolerance (generally taken as $10^{-10}$ - see numerical applications). In Equation (27) the explicit form of $F_{\text {int }}^{n+(1 / 2)}$ was not given. In the subsequent section the final expression for $F_{\text {int }}^{n+(1 / 2)}$ will be tailored so that it verifies the conservation conditions.

\subsection{The conservation conditions}

Equation (27) has to verify the linear and angular momentum conservation, and the energy balance. The first two conditions result from the physical laws assuming that the internal forces cannot change the rigid motion of a body. The last condition assumes that the total energy of the system is preserved for a reversible transformation and that the total energy is decreasing for an irreversible transformation.

3.2.1. The linear momentum conservation. Let $L$ be the vector (first order tensor) discrete linear momentum

$$
L \equiv \sum_{\xi} M^{\xi \mu} \dot{x}^{\mu}
$$

where we have adopted the convention of summing on repeated indices. The conservation of $L$ over a time step is discretized to

$$
L^{n+1}-L^{n}=\Delta t \sum_{\xi} F_{\mathrm{ext}}^{n+(1 / 2)^{\xi}}
$$


By performing a sum on $\xi$ in Equation (27), and using (26), it leads to

$$
\begin{aligned}
\frac{1}{\Delta t} \sum_{\xi} M^{\xi} \mu\left[\dot{x}^{n+1}-\dot{x}^{n}\right]^{\mu} & =\sum_{\xi}\left[F_{\mathrm{ext}}^{n+(1 / 2)}-F_{\mathrm{int}}^{n+(1 / 2)}\right]^{\xi} \\
\frac{1}{\Delta t}\left[L^{n+1}-L^{n}\right] & =\sum_{\xi}\left[F_{\mathrm{ext}}^{n+(1 / 2)}-F_{\mathrm{int}}^{n+(1 / 2)}\right]^{\xi}
\end{aligned}
$$

If (34) is compared to (35), the internal forces have to verify the following relation:

$$
\sum_{\xi}\left[F_{\mathrm{int}}^{n+(1 / 2)}\right]^{\xi}=0
$$

3.2.2. The angular momentum conservation. Let $J$ be the first order tensor discrete angular momentum

$$
J \equiv M^{\xi \mu}\left[x^{\xi} \wedge \dot{x}^{\mu}\right]
$$

The conservation of $J$ over a time step is discretized in

$$
J^{n+1}-J^{n}=\Delta t\left[x^{n+(1 / 2)}\right]^{\xi} \wedge\left[F_{\mathrm{ext}}^{n+(1 / 2)}\right]^{\xi}
$$

The vector product of $x^{n+(1 / 2)}$ and of relation (27) gives

$$
M^{\xi \mu}\left[x^{n+(1 / 2)}\right]^{\xi} \wedge\left[\ddot{x}^{n+(1 / 2)}\right]^{\mu}=\left[x^{n+(1 / 2)}\right]^{\xi} \wedge\left[F_{\mathrm{ext}}^{n+(1 / 2)}-F_{\mathrm{int}}^{n+(1 / 2)}\right]^{\xi}
$$

Using relations (26), this last expression leads to

$$
\begin{aligned}
& \frac{1}{\Delta t} M^{\xi \mu}\left\{\left[x^{n+1}\right]^{\xi} \wedge\left[\dot{x}^{n+1}\right]^{\mu}-\left[x^{n}\right]^{\xi} \wedge\left[\dot{x}^{n}\right]^{\mu}\right\}=\left[x^{n+(1 / 2)}\right]^{\xi} \wedge\left[F_{\mathrm{ext}}^{n+(1 / 2)}-F_{\mathrm{int}}^{n+(1 / 2)}\right]^{\mu} \\
& \frac{1}{\Delta t}\left[J^{n+1}-J^{n}\right]=\left[x^{n+(1 / 2}\right]^{\xi} \wedge\left[F_{\mathrm{ext}}^{n+(1 / 2)}\right]^{\xi}-\left[x^{n+(1 / 2)}\right]^{\xi} \wedge\left[F_{\mathrm{int}}^{n+(1 / 2)}\right]^{\xi}
\end{aligned}
$$

If we compare (38) and (40), the internal forces must thus verify

$$
\left[x^{n+(1 / 2)^{\xi}} \wedge F_{\text {int }}^{n+(1 / 2)^{\xi}}\right]=0
$$

3.2.3. The energy balance. Let $E, W_{\text {int }}, W_{\text {ext }}$ and $K$, respectively, be the total energy, the internal energy, the external energy and the kinetic energy. Usually (spring, hyperelastic model) the internal energy could be defined by a potential. Nevertheless, for a hypoelastic model, no potential could be defined but we will sidestep this difficulty in the next section by using the work of the internal forces. It comes

$$
E=K+W_{\text {int }}-W_{\text {ext }}
$$

The energy balance over one time step is discretized in

$$
\begin{aligned}
E^{n+1}-E^{n} & =-\Delta_{\text {int }} \\
& \leqslant 0
\end{aligned}
$$


with $\Delta_{\text {int }}$ the dissipation during the time step from configuration $n$ to $n+1$. The scalar product of $\dot{x}^{n+(1 / 2)}$ and of relation (27) leads to (using relation (26))

$$
\begin{aligned}
M^{\xi} \mu\left[\ddot{x}^{n+(1 / 2)}\right]^{\mu} *\left[\dot{x}^{n+(1 / 2)}\right]^{\xi} & =\left[F_{\mathrm{ext}}^{n+(1 / 2)}-F_{\mathrm{int}}^{n+(1 / 2)}\right]^{\xi} *\left[\dot{x}^{n+(1 / 2)}\right]^{\xi} \\
\frac{M^{\xi \mu}}{2 \Delta t}\left\{\left[\dot{x}^{n+1}\right]^{\xi} *\left[\dot{x}^{n+1}\right]^{\mu}-\left[\dot{x}^{n}\right]^{\xi} *\left[\dot{x}^{n}\right]^{\mu}\right\} & =\frac{1}{\Delta t}\left[F_{\mathrm{ext}}^{n+(1 / 2)}-F_{\mathrm{int}}^{n+(1 / 2)}\right]^{\xi} *\left[x^{n+1}-x^{n}\right]^{\xi} \\
K^{n+1}-K^{n}+\left[F_{\mathrm{int}}^{n+(1 / 2)}\right]^{\xi} *\left[x^{n+1}-x^{n}\right]^{\xi} & =W_{\mathrm{ext}}^{n+1}-W_{\mathrm{ext}}^{n}
\end{aligned}
$$

If (43) is compared to (44), then the relation that the internal forces have to verify is

$$
\left[F_{\text {int }}^{n+(1 / 2)}\right]^{\xi} *\left[x^{n+1}-x^{n}\right]^{\xi}=W_{\text {int }}^{n+1}-W_{\text {int }}^{n}+\Delta_{\text {int }}
$$

The next section of this paper will propose a formulation of the internal forces $\left(F^{n+(1 / 2)}\right.$ depending on $x^{n}$ and $x^{n+1}$ ) for hypoelastic materials. We will prove that this expression verifies relations (36), (41) and (45).

\section{INTERNAL FORCES EXPRESSION FOR HYPOELASTIC MATERIALS}

The expression of the internal forces in the configuration $n$ is given by relation (25). Nevertheless, if this expression is evaluated for $x^{n+(1 / 2)}$, relation (45) is generally not verified. Moreover, the volume will then be evaluated in an intermediate configuration that will introduce a parasitic volume change (for example, in a rigid body motion, $\operatorname{det}\left[\mathbf{F}_{0}^{n+(1 / 2)}\right] \neq \frac{1}{2} \operatorname{det}\left[\mathbf{F}_{0}^{n+1}+\mathbf{F}_{0}^{n}\right]$ ). Therefore, the following expression for $\left[F_{\mathrm{int}}^{n+(1 / 2)}\right]$ is proposed

$$
\begin{aligned}
{\left[F_{\text {int }}^{n+(1 / 2)}\right]^{\xi} } & =\frac{1}{4} \int_{V_{0}}\left\{\left[\mathbf{I}+\mathbf{F}_{n}^{n+1}\right] \boldsymbol{\Sigma}^{n \mathrm{~T}} \mathbf{f}_{0}^{n \mathrm{~T}} D^{\xi} J_{0}^{n}+\left[\mathbf{I}+\mathbf{f}_{n}^{n+1}\right] \boldsymbol{\Sigma}^{n+1}{ }^{\mathrm{T}} \mathbf{f}_{0}^{n+1^{\mathrm{T}}} D^{\xi} J_{0}^{n+1}\right\} \mathrm{d} V_{0} \\
& =\frac{1}{2}\left[F_{\text {int }}^{*}+F_{\text {int }}^{* *}\right]^{\xi} \\
{\left[F_{\text {int }}^{*}\right]^{\xi} } & =\frac{1}{2} \int_{V_{0}}\left\{\left[\mathbf{I}+\mathbf{F}_{n}^{n+1}\right] \boldsymbol{\Sigma}^{n \mathrm{~T}} \mathbf{f}_{0}^{n \mathrm{~T}} D^{\xi} J_{0}^{n}\right\} \mathrm{d} V_{0} \\
{\left[F_{\text {int }}^{* *}\right]^{\xi} } & =\frac{1}{2} \int_{V_{0}}\left\{\left[\mathbf{I}+\mathbf{f}_{n}^{n+1}\right] \mathbf{\Sigma}^{n+1{ }^{\mathrm{T}}} \mathbf{f}_{0}^{n+1^{\mathrm{T}}} D^{\xi} J_{0}^{n+1}\right\} \mathrm{d} V_{0}
\end{aligned}
$$

The stress tensors are evaluated by the final rotation scheme combined with the radial return mapping (see Section 2.2). The stress tensor in configuration $n+1$ is evaluated from the stress tensor in configuration $n$. Therefore, the scheme remains incrementally objective. Moreover, in relation (46), the stress tensors are always integrated over their relative volume (through $J$ ). Therefore, no parasitic volume variation are induced. The tangent stiffness matrix corresponding to this expression of the internal forces is given in Appendix A.

In the next section, we will demonstrate that relation (46) verifies the conservation laws. Next, this expression of the internal forces is compared with the expression given by Simo and Tarnow [12]. 


\subsection{Verification of conservation laws}

The conservation of the linear and angular momentum is ensured by verifying respectively relations (36) and (41). The conservation of energy is ensured by verifying (45).

4.1.1. Linear momentum conservation. Relation (36) is directly verified by performing an addition over $\xi$ in Equation (46) and by using the following properties of the shape functions $(\forall K \in[1,3])$ :

$$
\begin{aligned}
\sum_{\xi} D_{K}^{\xi} & =\sum_{\xi} \frac{\partial \varphi^{\xi}}{\partial x_{K}^{0}} \\
& =0
\end{aligned}
$$

4.1.2. Angular momentum conservation. We will verify that $\left(F_{\text {int }}^{*}\right)_{i}^{\xi}$ and $\left(F_{\text {int }}^{* *}\right)_{i}^{\xi}$ from relation (46) both verify relation (41). Let $\varepsilon$ be the third order permutation tensor such that, for each vector $a$ and $b$, it comes $(a \wedge b)=\varepsilon:[a \otimes b]$, with the operation $[a \otimes b]_{i j}=a_{i} b_{j}$. Therefore, it leads to

$$
\begin{aligned}
2 x^{n+(1 / 2)^{\xi}} \wedge F_{\text {int }}^{* \xi} & =\varepsilon:\left\{\left[x^{n+1}{ }^{\xi}+x^{n \xi}\right] \otimes F_{\text {int }}^{*}\right\} \\
& =\frac{1}{2} \varepsilon:\left\{\left[x^{n+1 \xi}+x^{n \xi}\right] \otimes \int_{V_{0}}\left\{\left[\mathbf{I}+\mathbf{F}_{n}^{n+1}\right] \mathbf{\Sigma}^{n \mathrm{~T}} \mathbf{f}_{0}^{n+1^{\mathrm{T}}} D^{\xi} J_{0}^{n}\right\} \mathrm{d} V_{0}\right\}
\end{aligned}
$$

Using (2) and (4) yields

$$
\begin{aligned}
{\left[x^{n}\right]^{\xi} \otimes\left[\mathbf{f}_{0}^{n \mathrm{~T}} D^{\xi}\right] } & =\mathbf{I} \\
{\left[x^{n+1}\right]^{\xi} \otimes\left[\mathbf{f}_{0}^{n \mathrm{~T}} D^{\xi}\right] } & =\mathbf{F}_{n}^{n+1^{\mathrm{T}}}
\end{aligned}
$$

Thanks to relations (49) and the fact that $\boldsymbol{\Sigma}$ is symmetric, relation (48) becomes

$$
\begin{aligned}
4\left[x^{n+(1 / 2)}\right]^{\xi} \wedge\left[F_{\text {int }}^{*}\right]^{\xi} & =\varepsilon: \int_{V_{0}}\left\{\left[\mathbf{I}+\mathbf{F}_{n}^{n+1}\right] \mathbf{\Sigma}^{n}\left[\mathbf{I}+\mathbf{F}_{n}^{n+1}\right]^{\mathrm{T}} J_{0}^{n}\right\} \mathrm{d} V_{0} \\
& =\int_{V_{0}}\left\{\varepsilon: \boldsymbol{\Theta} J_{0}^{n}\right\} \mathrm{d} V_{0} \\
& =0
\end{aligned}
$$

This can be easily verified since $\boldsymbol{\Theta}=\left[\mathbf{I}+\mathbf{F}_{n}^{n+1}\right] \boldsymbol{\Sigma}^{n}\left[\mathbf{I}+\mathbf{F}_{n}^{n+1}\right]^{\mathrm{T}}$ is a symmetric tensor and $\varepsilon$ is an anti-symmetric tensor. Therefore $\varepsilon: \Theta$ is equal to zero. The same process with $F_{\text {int }}^{* *}$ also leads to

$$
\left[x^{n+(1 / 2)}\right]^{\xi} \wedge\left[F_{\text {int }}^{* *}\right]^{\xi}=0
$$

and Equation (41) is thus verified. 
4.1.3. Energy conservation. The energy balance is verified through relation (45). First $F_{\text {int }}^{*}$ (46) is included in (45). Using (2) and (4), it comes

$$
\begin{aligned}
{\left[x^{n+1}-x^{n}\right]^{\xi} *\left[F_{\text {int }}^{*}\right]^{\xi} } & =\frac{1}{2}\left[x^{n+1}-x^{n}\right]^{\xi} * \int_{V_{0}}\left\{\left[\mathbf{I}+\mathbf{F}_{n}^{n+1}\right] \boldsymbol{\Sigma}^{n} \mathbf{f}_{0}^{n \mathrm{~T}} D^{\xi} J_{0}^{n}\right\} \mathrm{d} V_{0} \\
& =\frac{1}{2} \int_{V_{0}}\left\{\left[\mathbf{F}_{n}^{n+1} \mathbf{F}_{n}^{n+1}+\mathbf{F}_{n}^{n+1}-\mathbf{F}_{n}^{n+1 \mathrm{~T}}-\mathbf{I}\right]: \boldsymbol{\Sigma}^{n} J_{0}^{n}\right\} \mathrm{d} V_{0}
\end{aligned}
$$

Since $\boldsymbol{\Sigma}$ is symmetric, it leads to

$$
\mathbf{F}_{n}^{n+1^{\mathrm{T}}}: \Sigma^{n}-\mathbf{F}_{n}^{n+1}: \Sigma^{n}=0
$$

Using (7) and (53), relation (52) becomes

$$
\left[x^{n+1}-x^{n}\right]^{\xi} *\left[F_{\text {int }}^{*}\right]^{\xi}=\int_{V_{0}}\left\{\mathbf{G L}_{n}^{n+1}: \boldsymbol{\Sigma}^{n} J_{0}^{n}\right\} \mathrm{d} V_{0}
$$

For $F_{\text {int }}^{* * \xi}$ the same process leads to

$$
\left[x^{n+1}-x^{n}\right]^{\xi} *\left[F_{\text {int }}^{* *}\right]^{\xi}=\int_{V_{0}}\left\{\mathbf{A}_{n}^{n+1}: \boldsymbol{\Sigma}^{n+1} J_{0}^{n+1}\right\} \mathrm{d} V_{0}
$$

and finally one gets

$$
\left[F_{\text {int }}^{n+(1 / 2)}\right]^{\xi} *\left[x^{n+1}-x^{n}\right]^{\xi}=\frac{1}{2} \int_{V_{0}}\left\{\mathbf{G L}_{n}^{n+1}: \boldsymbol{\Sigma}^{n} J_{0}^{n}+\mathbf{A}_{n}^{n+1}: \boldsymbol{\Sigma}^{n+1} J_{0}^{n+1}\right\} \mathrm{d} V_{0}
$$

which should be equal to $W_{\text {int }}^{n+1}-W_{\text {int }}^{n}+\Delta_{\text {int }}$ if the energy is conserved (45). From this point, for a hyperelastic material, a potential $\phi$ could be written to evaluate the internal energy [23]. However, for a hypoelastic material, no potential can be defined. Thus we will proceed differently in order to demonstrate (56). Let's imagine a loading-unloading cycle, that takes place in two steps, from configurations 1 to 3 (Figure 1), such that the initial Cauchy stress tensor $\boldsymbol{\Sigma}^{1}$ corresponds to the final Cauchy stress tensor $\boldsymbol{\Sigma}^{3}$ up to any arbitrary rotation $\mathbf{Q}$ $\left(\mathbf{Q}^{\mathrm{T}} \mathbf{Q}=\mathbf{I}\right.$ and $\left.\operatorname{det} \mathbf{Q}=1\right)$

$$
\Sigma^{3}=\mathbf{Q} \Sigma^{1} \mathbf{Q}^{\mathrm{T}}
$$

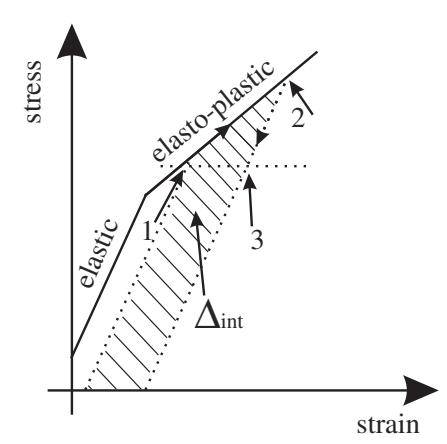

Figure 1. Definition of the loading-unloading cycle (1D analogy). 
During the loading phase from configurations 1 to 2 , we assume that plastic deformations occur, while the transition from configurations 2 to 3 corresponds to elastic unloading. Note that configuration 3 might be kinematically inadmissible for a whole body, but this is not of concern here since we are reasoning at the particle level. The expression of internal forces (46) is consistent with the Druckers Postulate (see e.g. Reference [49]) if the reversible work of the loading phase is recovered during the second step (i.e. $W_{\text {int }}^{3}-W_{\text {int }}^{1}=0$ ). Therefore, the energy balance between the configurations 1 and 3 can be expressed as

$$
\left[F_{\text {int }}^{(3 / 2)}\right]^{\xi} *\left[x^{2}-x^{1}\right]^{\xi}+\left[F_{\text {int }}^{(5 / 2)}\right]^{\xi} *\left[x^{3}-x^{2}\right]^{\xi}=\Delta_{\text {int }}
$$

Using relation (56) and relation (57), expression (58) becomes

$$
\begin{aligned}
\Delta_{\text {int }} & =\frac{1}{2} \int_{V_{0}}\left\{\mathbf{G L}_{1}^{2}: \boldsymbol{\Sigma}^{1} J_{0}^{1}+\mathbf{A}_{1}^{2}: \boldsymbol{\Sigma}^{2} J_{0}^{2}+\mathbf{G L}_{2}^{3}: \boldsymbol{\Sigma}^{2} J_{0}^{2}+\mathbf{A}_{2}^{3}:\left[\mathbf{Q} \boldsymbol{\Sigma}^{1} \mathbf{Q}^{\mathrm{T}}\right] J_{0}^{3}\right\} \mathrm{d} V_{0} \\
& =\frac{1}{2} \int_{V^{0}}\left\{J_{0}^{1} \mathbf{G} \mathbf{L}_{1}^{2}: \boldsymbol{\Sigma}^{1}+J_{0}^{3}\left[\mathbf{Q}^{\mathrm{T}} \mathbf{A}_{2}^{3} \mathbf{Q}\right]: \boldsymbol{\Sigma}^{1}+J^{0,2}\left[\mathbf{A}_{1}^{2}+\mathbf{G L}_{2}^{3}\right]: \boldsymbol{\Sigma}^{2}\right\} \mathrm{d} V_{0}
\end{aligned}
$$

Now we examine the implications of relation (57). Let $\mathbf{E}_{1}^{\mathrm{e}^{2}}$ be the elastic natural strain tensor defined such that

$$
\mathscr{H}: \mathbf{E}_{1}^{\mathrm{e}^{2}} \equiv \mathscr{H}: \mathbf{E}_{1}^{2}-\mathbf{s}_{1}^{c^{2}}
$$

It yields from Equation (15)

$$
\begin{aligned}
\boldsymbol{\Sigma}^{2} & =\mathbf{R}_{1}^{2}\left[\boldsymbol{\Sigma}^{1}+\mathscr{H}: \mathbf{E}_{1}^{2}-\mathbf{s}_{1}^{c^{2}}\right] \mathbf{R}_{1}^{2^{\mathrm{T}}} \\
& =\mathbf{R}_{1}^{2}\left[\boldsymbol{\Sigma}^{1}+\mathscr{H}: \mathbf{E}_{1}^{\mathrm{e}^{2}}\right] \mathbf{R}_{1}^{2^{\mathrm{T}}}
\end{aligned}
$$

Therefore we define $\mathbf{U}_{1}^{\mathrm{el}^{2}}$ the symmetric tensor such that

$$
\mathbf{E}_{1}^{\mathrm{el}^{2}} \equiv \frac{1}{2} \ln \left[\mathbf{U}_{1}^{\mathrm{el}^{2}} \mathbf{U}_{1}^{\mathrm{el}^{2}}\right]
$$

The existence of $\mathbf{U}_{1}^{\mathrm{e}^{2}}$ result from the symmetry of tensor $\mathbf{E}_{1}^{\mathrm{e}^{2}}$. The elastic Green-Lagrange strain tensor $\mathbf{G L}_{1}^{\mathrm{el}^{2}}$, and the elastic Almansi strain tensor $\mathbf{A}_{1}^{\mathrm{el}^{2}}$ are defined from $\mathbf{U}_{1}^{\mathrm{el}^{2}}$

$$
\begin{aligned}
\mathbf{G L}_{1}^{\mathrm{el}^{2}} & \equiv \frac{1}{2}\left[\mathbf{U}_{1}^{\mathrm{el}^{2}} \mathbf{U}_{1}^{\mathrm{e}^{2}}-\mathbf{I}\right] \\
\mathbf{A}_{1}^{\mathrm{el}^{2}} & \equiv \frac{1}{2} \mathbf{R}_{1}^{2}\left[\mathbf{I}-\mathbf{U}_{1}^{\mathrm{el}^{2^{-1}}} \mathbf{U}_{1}^{\mathrm{e}^{2^{-1}}}\right] \mathbf{R}_{1}^{2^{\mathrm{T}}}
\end{aligned}
$$

Finally, the corresponding plastic tensors can be defined as

$$
\begin{aligned}
\mathbf{G L}_{1}^{\mathrm{pl}} & \equiv \mathbf{G L}_{1}^{2}-\mathbf{G L}_{1}^{\mathrm{el}^{2}} \\
\mathbf{A}_{1}^{\mathrm{pl}^{2}} & \equiv \mathbf{A}_{1}^{2}-\mathbf{A}_{1}^{\mathrm{el}^{2}}
\end{aligned}
$$


Now we must compute the variables in configuration 3 from these defined values. Using relations (57) yields

$$
\begin{aligned}
\mathbf{Q} \boldsymbol{\Sigma}^{1} \mathbf{Q}^{\mathrm{T}} & =\boldsymbol{\Sigma}^{3} \\
& =\mathbf{R}_{2}^{3}\left[\mathbf{R}_{1}^{2} \boldsymbol{\Sigma}^{1} \mathbf{R}_{1}^{2^{\mathrm{T}}}+\mathbf{R}_{1}^{2} \mathscr{H}: \mathbf{E}_{1}^{\mathrm{e} 1^{2}} \mathbf{R}_{1}^{2^{\mathrm{T}}}+\mathscr{H}: \mathbf{E}_{2}^{3}\right] \mathbf{R}_{2}^{3^{\mathrm{T}}} \\
& =\mathbf{R}_{2}^{3} \mathbf{R}_{1}^{2} \boldsymbol{\Sigma}^{1} \mathbf{R}_{1}^{2^{\mathrm{T}}} \mathbf{R}_{2}^{3^{\mathrm{T}}}+\mathbf{R}_{2}^{3} \mathbf{R}_{1}^{2} \mathscr{H}: \mathbf{E}_{1}^{\mathrm{e}^{2}} \mathbf{R}_{1}^{2^{\mathrm{T}}} \mathbf{R}_{2}^{3^{\mathrm{T}}}+\mathbf{R}_{2}^{3} \mathscr{H}: \mathbf{E}_{2}^{3} \mathbf{R}_{2}^{3^{\mathrm{T}}}
\end{aligned}
$$

From this relation, assuming that $\mathscr{H}$ is constant between configurations 1 and 3 , we can see that the transformation from configurations 2 to 3 must have the following properties:

$$
\mathbf{R}_{2}^{3}=\mathbf{Q R}_{1}^{2^{\mathrm{T}}}
$$

and

$$
\mathscr{H}: \mathbf{E}_{2}^{3}=-\mathbf{R}_{1}^{2} \mathscr{H}: \mathbf{E}_{1}^{\mathrm{el}^{2}} \mathbf{R}_{1}^{2^{\mathrm{T}}}
$$

in order to be consistent with our definition of configuration 3. Using relations (5), (9), (14), (63) and (67), yields

$$
\begin{aligned}
\mathbf{G L}_{2}^{3} & =-\mathbf{A}_{1}^{\mathrm{e}^{2}} \\
\mathbf{A}_{2}^{3} & =-\mathbf{Q G L}_{1}^{\mathrm{el}^{2}} \mathbf{Q}^{\mathrm{T}}
\end{aligned}
$$

From relation (67), we have

$$
\begin{aligned}
{\left[\mathscr{H}: \mathbf{E}_{2}^{3}\right]_{i i} } & =\left[-\mathbf{R}_{1}^{2} \mathscr{H}: \mathbf{E}_{1}^{\mathrm{el}^{2}} \mathbf{R}_{1}^{2^{\mathrm{T}}}\right]_{i i} \\
{\left[\mathscr{H}: \mathbf{E}_{2}^{3}\right]_{i i} } & =\left[-\mathscr{H}: \mathbf{E}_{1}^{\mathrm{e}^{2}}\right]_{i i} \\
3 k\left[\mathbf{E}_{2}^{3}\right]_{i i} & =3 k\left[-\mathbf{E}_{1}^{\mathrm{el}^{2}}\right]_{i i}
\end{aligned}
$$

and, since the trace of a logarithm mapping corresponds to the logarithm of the determinant of the matrix, we have the determinant of $\mathbf{U}_{1}^{\mathrm{e}^{2}}$ equal to the inverse of the determinant of $\mathbf{U}_{2}^{3}$. Using the same technique from relation (60), and since the tensor $\mathbf{s}^{\mathrm{c}}$ is trace less, the determinant of $\mathbf{U}_{1}^{\mathrm{el}^{2}}$ is equal to the determinant of $\mathbf{U}_{1}^{2}$, and it leads to

$$
J_{0}^{3}=J_{0}^{1}
$$

Therefore, using relations (68) and (70), relation (59) becomes

$$
\begin{aligned}
\Delta_{\text {int }} & =\frac{1}{2} \int_{V^{0}}\left\{\left[\mathbf{G L}_{1}^{2}-\mathbf{G L}_{1}^{\left.\mathrm{e}\right|^{2}}\right]: \boldsymbol{\Sigma}^{1} J^{0,1}+\left[\mathbf{A}_{1}^{2}-\mathbf{A}_{1}^{\left.\mathrm{e}\right|^{2}}\right]: \boldsymbol{\Sigma}^{2} J^{0,2}\right\} \mathrm{d} V_{0} \\
& =\frac{1}{2} \int_{V^{0}}\left\{\mathbf{G L}_{1}^{\mathrm{p} 1^{2}}: \boldsymbol{\Sigma}^{1} J^{0,1}+\mathbf{A}_{1}^{\mathrm{p} l^{2}}: \boldsymbol{\Sigma}^{2} J^{0,2}\right\} \mathrm{d} V_{0}
\end{aligned}
$$

If there is no plastic strain increment between configuration 1 and $2, \mathbf{G L}_{1}^{\mathrm{el}^{2}}$ and $\mathbf{A}_{1}^{\mathrm{el}^{2}}$ are respectively equal to $\mathbf{G L}_{1}{ }_{1}$ and $\mathbf{A}_{1}{ }^{2}$. Therefore, $\Delta_{\text {int }}$ is also equal to zero. This verifies the laws 
of thermodynamics. On the other hand, if there is plastic strain increment, the relation (71) has to be related with a physical relation that is positive. The internal plastic dissipation can be expressed from a volumic dissipation $D_{\text {int }}$ as

$$
\begin{aligned}
\Delta_{\text {int }}^{\text {phy }} & =\int_{V_{0}}\left\{D_{\text {int }}\right\} \mathrm{d} V_{0} \\
& >0
\end{aligned}
$$

Nevertheless, the equivalence between $\Delta_{\text {int }}$ and $\Delta_{\text {int }}^{\text {phy }}$ does not exist. So we introduce two corrections $\left(\mathbf{c}^{*}\right.$ and $\left.\mathbf{c}^{* *}\right)$ in the evaluation of the internal forces (46)

$$
\begin{aligned}
{\left[F_{\text {int }}^{*}\right]^{\xi} } & =\frac{1}{2} \int_{V_{0}}\left\{\left[\mathbf{I}+\mathbf{F}_{n}^{n+1}\right]\left[\boldsymbol{\Sigma}^{n}+\frac{\mathbf{c}^{*}: \mathbf{\Sigma}^{n}}{\mathbf{G L}_{n}^{n+1}: \mathbf{G L}_{n}^{n+1}} \mathbf{G L}_{n}^{n+1}\right]^{\mathrm{T}} \mathbf{f}_{0}^{n \mathrm{~T}} D^{\xi} J_{0}^{n}\right\} \mathrm{d} V_{0} \\
{\left[F_{\text {int }}^{* *}\right]^{\xi} } & =\frac{1}{2} \int_{V_{0}}\left\{\left[\mathbf{I}+\mathbf{f}_{n}^{n+1}\right]\left[\boldsymbol{\Sigma}^{n+1}+\frac{\mathbf{c}^{* *}: \boldsymbol{\Sigma}^{n+1}}{\mathbf{A}_{n}^{n+1}: \mathbf{A}_{n}^{n+1}} \mathbf{A}_{n}^{n+1}\right]^{\mathrm{T}} \mathbf{f}_{0}^{n+1} D^{\mathrm{T}} D_{0}^{\xi} J_{0}^{n+1}\right\} \mathrm{d} V_{0}
\end{aligned}
$$

where $\mathbf{c}^{*}$ and $\mathbf{c}^{* *}$ are tensors to be determined. The conservation of the linear momentum is not affected by these corrections (relation 47), and since the corrections are symmetrical tensors, the conservation of the angular momentum remains verified (relations 50 and 51). On the other hand, the expression (71) of the internal dissipation is modified. Assuming that the correcting tensors are to be equal to zero when no plastic deformation occurs (i.e. during the transformation from configurations 2 to 3 ) yields to

$$
\Delta_{\text {int }}=\frac{1}{2} \int_{V^{0}}\left\{\left[\mathbf{G L}_{n}^{\mathrm{pl}^{n+1}}+\mathbf{c}^{*}\right]: \boldsymbol{\Sigma}^{n} J^{0, n}+\left[\mathbf{A}_{n}^{\mathrm{p}^{n+1}}+\mathbf{c}^{* *}\right]: \boldsymbol{\Sigma}^{n+1} J^{0, n+1}\right\} \mathrm{d} V_{0}
$$

With the tensors

$$
\begin{aligned}
\mathbf{c}^{*} & =\frac{D_{\text {int }}}{\boldsymbol{\Sigma}^{n} J_{0}^{n}: \boldsymbol{\Sigma}^{n+1} J_{0}^{n+1}} \boldsymbol{\Sigma}^{n+1} J_{0}^{n+1}-\mathbf{G L}_{n}^{n+1}+\mathbf{G L}_{n}{ }^{\mathrm{el}^{n+1}} \\
\mathbf{c}^{* *} & =\frac{D_{\text {int }}}{\boldsymbol{\Sigma}^{n} J_{0}^{n}: \boldsymbol{\Sigma}^{n+1} J_{0}^{n+1}} \boldsymbol{\Sigma}^{n} J_{0}^{n}-\mathbf{A}_{n}^{n+1}+\mathbf{A}_{n}^{\mathrm{e}^{n+1}}
\end{aligned}
$$

relation (74) becomes (using relation (72) and the fact that the physical dissipation is positive)

$$
\begin{aligned}
\Delta_{\text {int }} & =\Delta_{\text {int }}^{\text {phy }} \\
& >0
\end{aligned}
$$

The order of these correction tensors is now determined. Relation (60) can be transformed, using relations (9) and (17) as

$$
\begin{aligned}
\mathscr{H}: \mathbf{E}_{n}^{\mathrm{e}^{n+1}} & =\mathscr{H}: \mathbf{E}_{n}^{n+1}-\mathbf{s}_{n}^{c^{n+1}} \\
\mathscr{H}: \mathbf{G L}_{n}^{\mathrm{e}^{n+1}} & =\mathscr{H}: \mathbf{G L}_{n}^{n+1}+\mathcal{O}\left(\mathbf{G L}_{n}^{\mathrm{pl}^{n+1^{2}}}\right)-2 g \gamma \mathbf{N}
\end{aligned}
$$


or as (with $\mathbf{N}^{r}=\mathbf{R}_{n}^{n+1} \mathbf{N R}_{n}^{n+1^{\mathrm{T}}}$ )

$$
\mathscr{H}: \mathbf{A}_{n}^{\left.\mathrm{e}\right|^{n+1}}=\mathscr{H}: \mathbf{A}_{n}^{n+1}+\mathcal{O}\left(\mathbf{A}_{n}^{\mathrm{p}^{n+1^{2}}}\right)-2 g \gamma \mathbf{N}^{r}
$$

Using the fact that for $J 2$ plasticity the trace of $\mathbf{N}$ is equal to zero, the inversion of the Hooke law (relation 14) yields

$$
\begin{aligned}
\mathbf{G L}_{n}^{n+1}-\mathbf{G L}_{n}^{\mathrm{e}^{n+1}}+\mathcal{O}\left(\mathbf{G} \mathbf{L}_{n}^{\mathrm{p}^{n+1^{2}}}\right) & =\gamma \mathbf{N} \\
\mathbf{A}_{n}^{n+1}-\mathbf{A}_{n}^{\mathrm{e} l^{n+1}}+\mathcal{O}\left(\mathbf{A}_{n}^{\mathrm{pl}^{n+1^{2}}}\right) & =\gamma \mathbf{N}^{r}
\end{aligned}
$$

Therefore, the order of relations (75) is

$$
\begin{gathered}
\mathbf{c}^{*}: \boldsymbol{\Sigma}^{n} J_{0}^{n}=D_{\mathrm{int}}-\gamma \mathbf{N}: \boldsymbol{\Sigma}^{n} J_{0}^{n}+\mathcal{O}\left(\boldsymbol{\Sigma}^{n}: \mathbf{G} \mathbf{L}_{n}{ }^{\mathrm{p}^{n+1^{2}}}\right) \\
\mathbf{c}^{* *}: \boldsymbol{\Sigma}^{n+1} J_{0}^{n+1}=D_{\mathrm{int}}-\gamma \mathbf{N}^{r}: \boldsymbol{\Sigma}^{n+1} J_{0}^{n+1}+\mathcal{O}\left(\boldsymbol{\Sigma}^{n+1}: \mathbf{A}_{n}^{\mathrm{p}^{n+1^{2}}}\right)
\end{gathered}
$$

The internal dissipation can be expressed as

$$
\begin{aligned}
D_{\text {int }} & =\frac{1}{2} \varepsilon_{n}^{\mathrm{p} n+1}\left\{\left[\Sigma_{v}{ }^{n+1}+\bar{\alpha}^{n+1}\right] J_{0}^{n+1}+\left[\sum_{v}^{n}+\bar{\alpha}^{n}\right] J_{0}^{n}\right\} \\
& >0
\end{aligned}
$$

With the definition of $\gamma(17), \varepsilon(17), \mathbf{N}(16)$ and $\mathbf{s}^{n}$ defining the deviatoric part of $\boldsymbol{\Sigma}^{n}$, the relation (18), at the first order leads

$$
\begin{aligned}
\gamma \mathbf{N}: \Sigma^{n} J_{0}^{n} & =\gamma \mathbf{N}: \mathbf{s}^{n} J_{0}^{n} \\
& \simeq \varepsilon^{\mathrm{p} n+1}\left[\Sigma_{v}^{n}+\bar{\alpha}^{n}\right] J_{0}^{n} \\
\gamma \mathbf{N}: \Sigma^{n+1} J_{0}^{n+1} & =\gamma \mathbf{N}^{r}: \mathbf{s}^{n+1} J_{0}^{n+1} \\
& \simeq \varepsilon^{\mathrm{p} n+1}\left[\Sigma_{v}{ }^{n+1}+\bar{\alpha}^{n+1}\right] J_{0}^{n+1}
\end{aligned}
$$

And finally, the sum of the two term of expression (80) leads to second order terms. Therefore, for small increments of transformation, the correction tensors are of the second order. In this paper, the time steps sizes are taken small enough so that the correcting tensors can be neglected. In a further work, we will prove that if the time step is increased (and therefore the plastic strain increment) these correcting tensors must be taken into account unless the relation (76) is not longer verified and the dissipation could even be positive.

\section{Remark}

For one hypoelastic model, the stress could exhibit oscillations for a load-unload cycle over some time steps. Nevertheless, this results from the model and not from the proposed method since the demonstration is based on the hypothesis that the loading-unloading is computed altogether during one single time step. Now, the proposed method is compared with other existing models. 
4.1.4. Small transformation hypothesis. If the total strain and the rotation are assumed to be small, we define the small strain (second order tensor) $\varepsilon=\frac{1}{2}\left(\partial u_{i} / \partial x_{j}+\partial u_{j} / \partial x_{i}\right)$ where $u$ is the small displacement vector and the Cauchy stress (second order tensor) $\sigma$. Therefore, it follows

$$
\begin{aligned}
J_{0}^{n+1} & \simeq J_{0}^{n} \\
\mathbf{G} \mathbf{L}_{n}^{\mathrm{el}^{n+1}} & \simeq \boldsymbol{\varepsilon}_{n}^{\mathrm{e}^{n+1}} \\
\mathbf{A}_{n}^{\mathrm{el}^{n+1}} & \simeq \boldsymbol{\varepsilon}_{n}^{\mathrm{e}^{n+1}} \\
\mathbf{E}_{n}^{\mathrm{e}^{n+1}} & \simeq \boldsymbol{\varepsilon}_{n}^{\mathrm{e}^{n+1}} \\
\mathbf{E}_{0}^{\mathrm{e}^{n+1}} & \simeq \mathbf{E}_{0}^{\mathrm{el}^{n}}+\mathbf{E}_{n}^{\mathrm{el}^{n+1}} \\
\mathbf{R}_{0}^{n} & \simeq \mathbf{I} \\
\boldsymbol{\sigma}^{n} & \simeq \mathscr{H}: \mathbf{E}_{0}^{\mathrm{el}^{n}} \\
\mathbf{G L}_{n}^{\mathrm{pl}^{n+1}} & \simeq \boldsymbol{\varepsilon}_{n}^{\mathrm{p}^{n+1}} \\
\mathbf{A}_{n}^{\mathrm{p}^{n+1}} & \simeq \boldsymbol{\varepsilon}_{n}^{\mathrm{p}^{n+1}} \\
\mathbf{G L}_{n}^{n+1} & \simeq \boldsymbol{\varepsilon}_{n}^{\mathrm{e}^{n+1}}+\boldsymbol{\varepsilon}_{n}^{\mathrm{p} \mathrm{p}^{n+1}} \\
\mathbf{A}_{n}^{n+1} & \simeq \boldsymbol{\varepsilon}_{n}^{\mathrm{e}^{n+1}}+\boldsymbol{\varepsilon}_{n}^{\mathrm{p}^{n+1}}
\end{aligned}
$$

The internal potential is defined by

$$
W_{\text {int }}^{n}=\frac{1}{2} \int_{V_{0}}\left\{\boldsymbol{\varepsilon}_{0}^{\mathrm{e}^{n}}: \mathscr{H}: \boldsymbol{\varepsilon}_{0}^{\mathrm{e}^{n}}\right\} \mathrm{d} V_{0}
$$

With the hypothesis expressed in (83), expression (56) becomes

$$
\begin{aligned}
{\left[F_{\text {int }}^{n+(1 / 2)}\right]^{\xi} *\left[x^{n+1}-x^{n}\right]^{\xi}=} & \frac{1}{2} \int_{V_{0}}\left\{\left[\varepsilon_{0}^{\mathrm{e}^{n+1}}-\boldsymbol{\varepsilon}_{0}^{\mathrm{e}^{n}}+\boldsymbol{\varepsilon}_{n}^{\mathrm{p}^{n+1}}\right]: \mathscr{H}: \mathbf{E}_{0}^{\mathrm{e}^{n}}\right\} \mathrm{d} V_{0} \\
& +\frac{1}{2} \int_{V_{0}}\left\{\left[\varepsilon_{0}^{\mathrm{e}^{n+1}}-\varepsilon_{0}^{\mathrm{e}^{n}}+\boldsymbol{\varepsilon}_{n}^{\mathrm{p}^{n+1}}\right): \mathscr{H}: \mathbf{E}_{0}^{\mathrm{e}^{n+1}}\right\} \mathrm{d} V_{0} \\
= & W_{\text {int }}^{n+1}-W_{\text {int }}^{n} \\
& +\frac{1}{2} \int_{V_{0}}\left\{\boldsymbol{\varepsilon}_{n}^{\mathrm{p}^{n+1}}: \boldsymbol{\sigma}^{n}+\boldsymbol{\varepsilon}_{n}^{\mathrm{p}^{n+1}}: \boldsymbol{\sigma}^{n+1}\right\} \mathrm{d} V_{0}
\end{aligned}
$$

This last expression corresponds to the usual definition of the internal reversible and irreversible work for models defined by an internal potential.

4.1.5. Comparison with the hyperelastic model. The conserving scheme for a hyperelastic material uses a flow definition to compute the plastic deformation [23]. The present paper 
uses a final rotation scheme combined with the radial return mapping to compute the plastic deformation. Since the plastic formulation for an hyperelastic material differs from the plastic formulation for a hypoelastic material, we consider the case where no plastic deformation occurs. The Cauchy stress tensor is transformed into the Piola-Kirchhoff stress tensor, using relation (10). Expression (46) is therefore rewritten as

$$
\begin{aligned}
{\left[F_{\text {int }}^{n+(1 / 2)}\right]^{\xi}=} & \frac{1}{4} \int_{V_{0}}\left\{\left[\mathbf{I}+\mathbf{F}_{n}^{n+1}\right] \mathbf{F}_{0}^{n} \mathbf{S}^{n} \mathbf{F}_{0}^{n \mathrm{~T}} \mathbf{f}_{0}^{n \mathrm{~T}} D^{\xi} J_{0}^{n}\right\} \mathrm{d} V_{0} \\
& +\frac{1}{4} \int_{V_{0}}\left\{\left[\mathbf{I}+\mathbf{f}_{n}^{n+1}\right] \mathbf{F}_{0}^{n+1} \mathbf{S}^{n+1} \mathbf{F}_{0}^{n+1} \mathbf{f}_{0}^{\mathrm{T}}{ }^{n+1} D^{\mathrm{T}} J_{0}^{n+1}\right\} \mathrm{d} V_{0} \\
= & \frac{1}{4} \int_{V_{0}}\left\{\left[\mathbf{F}_{0}^{n}+\mathbf{F}_{0}^{n+1}\right]\left[\mathbf{S}^{n}+\mathbf{S}^{n+1}\right] D^{\xi}\right\} \mathrm{d} V_{0}
\end{aligned}
$$

This last expression corresponds to the expression established by Simo and Tarnow [12] for the Saint Venant-Kirchhoff material. This similitude is consistent with the fact that the elastic part of the hypoelastic formulation has the same behavior as the Saint Venant-Kirchhoff formulation. Moreover, using relations (4), (8), (9) and (10), expression (56) can be rewritten as

$$
\begin{aligned}
W_{\text {int }}^{n+1}-W_{\text {int }}^{n}+\Delta_{\text {int }} & =\frac{1}{2} \int_{V_{0}}\left\{\mathbf{G} \mathbf{L}_{n}^{n+1}: \mathbf{\Sigma}^{n} J_{0}^{n}+\mathbf{A}_{n}^{n+1}: \boldsymbol{\Sigma}^{n+1} J_{0}^{n+1}\right\} \\
& =\frac{1}{2} \int_{V_{0}}\left\{\left[\mathbf{F}_{0}^{n \mathrm{~T}} \mathbf{G L}_{n}^{n+1} \mathbf{F}_{0}^{n}\right]: \mathbf{S}^{n}+\left[\mathbf{F}_{0}^{n+1} \mathbf{A}_{n}^{n+1} \mathbf{F}_{0}^{n+1}\right]: \mathbf{S}^{n+1}\right\} \mathrm{d} V_{0} \\
& =\frac{1}{2} \int_{V_{0}}\left\{\left[\mathbf{G L}_{0}^{n+1}-\mathbf{G L}_{0}^{n}\right]: \mathbf{S}^{n+(1 / 2)}\right\} \mathrm{d} V_{0}
\end{aligned}
$$

For a Saint Venant-Kirchhoff material, without plastic deformation, this latter expression is reduced to $\Delta_{\text {int }}=0$ [12].

\section{NUMERICAL EXAMPLES}

In this section the results obtained with the proposed conservative scheme (EMCA) are compared with the results obtained with:

(i) the Newmark algorithm [1] (NMK) with the first Newmark parameter $(\beta)$ equal to 0.25 and the second Newmark parameter $(\gamma)$ equal to 0.5 ;

(ii) the Chung-Hulbert algorithm [9] $(\mathrm{CH})$ with $\beta$ equal to $0.9801, \gamma$ equal to 1.48 , the inertial forces parameter $\left(\alpha_{\mathrm{M}}\right)$ equal to -0.97 and the internal forces parameter $\left(\alpha_{\mathrm{F}}\right)$ equal to 0.01 ;

(iii) the Hilbert-Hughes-Taylor algorithm [50] (HHT) with $\beta$ equal to $0.255025, \gamma$ equal to 0.51 and $\alpha$ equal to 0.05 . 
Table I. Properties of the beam in uniform rotation.

\begin{tabular}{lc}
\hline Property & Value \\
\hline Length & $L=1 \mathrm{~m}$ \\
Width & $l=0.01 \mathrm{~m}$ \\
Density & $\rho=4000 \mathrm{~kg} / \mathrm{m}^{3}$ \\
Young's modulus & $E=10^{11} \mathrm{~N} / \mathrm{m}^{2}$ \\
Poisson ratio & $\nu=0.3$ \\
Initial angular velocity & $\Omega=2909 \mathrm{rpm}$ \\
\hline
\end{tabular}

For the hypoelastic material, the internal energy is not directly accessible. Therefore, the total energy is computed from the work of internal forces. For the conservative algorithm, the total energy at time $t_{n+1}$ is defined as

$$
E^{n+1}=E^{n}+K^{n+1}-K^{n}+\left[F_{\text {int }}^{n+(1 / 2)}\right]\left[x^{n+1}-x^{n}\right]
$$

with $F_{\text {int }}^{n+(1 / 2)}$ computed from relation (46). For the other algorithms, it is defined by

$$
E^{n+1}=E^{n}+K^{n+1}-K^{n}+\frac{1}{2}\left[F_{\text {int }}^{n}+F_{\text {int }}^{n+1}\right]\left[x^{n+1}-x^{n}\right]
$$

with $F_{\text {int }}^{n}$ defined from relation (25). This total energy evaluation includes the internal dissipation and must thus remain constant for each problem. Therefore, the variation of the energy comes only from the numerical modes. The internal dissipation is evaluated from relation (71) for each algorithm. This relation was established for the conservative scheme but remains physically correct for the other algorithms.

The finite elements used for each example are eight-noded trilinear bricks with eight deviatoric Gauss points and one volumic Gauss point. For each problem, the time step size is constant to avoid any instabilities resulting from a time step size variation, and is chosen small enough to avoid the obligation of taking into account the terms of the second order in the increment of the plastic deformation. Let us note that the total plastic deformation is not limited by this restriction. Moreover the tolerance on the residual (32) is set to $10^{-10}$ for each problem and for each algorithm.

\subsection{Example 1: The uniform rotation of a beam}

This problem consists of a beam with a constant square section. Its properties are given in Table I. This beam has an initial angular velocity $\Omega$ (Table I). The material of the beam is assumed elastic. The mesh consists of 48 elements. The median nodes of one of the extremities belong to the rotation axis and are fixed (no displacement, but rotation is allowed). The initial balanced configuration is computed with a Newton-Raphson algorithm where the inertial (centrifugal) forces are computed analytically. This balanced configuration is illustrated in Figure 2. The time step size is equal to $0.5 \mathrm{~ms}$.

Figures 3 and 4 represent the evolution of the angular momentum. Only the conservative scheme leads to a constant value. The evolution of the total energy is reported in Figures 5 and 6. The conservative scheme preserves the total energy. For the Chung-Hulbert and the Hilbert-Hughes-Taylor schemes, numerical dissipation occurs (4\% of the total energy is lost in a round for the Chung-Hulbert scheme and $0.03 \%$ of the total energy is lost in a round 


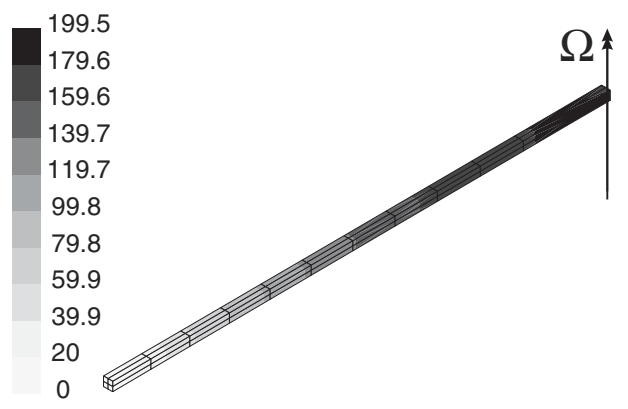

Figure 2. Initial configuration and von Mises stress $\left(\mathrm{N} / \mathrm{mm}^{2}\right)$ for the beam in uniform rotation.

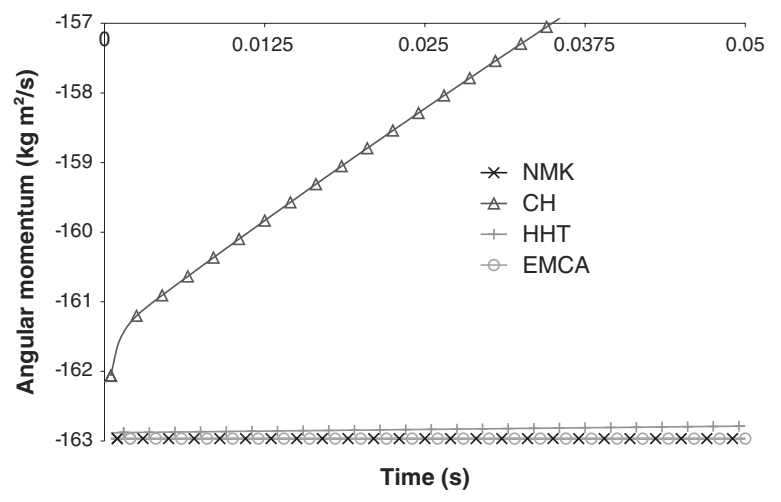

Figure 3. Angular momentum evolution for the beam in uniform rotation.

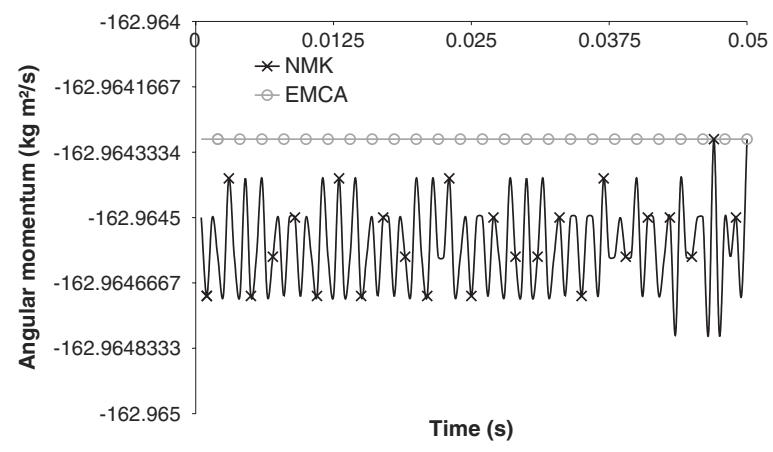

Figure 4. Angular momentum evolution for the beam in uniform rotation (zoom).

for the Hilbert-Hughes-Taylor scheme). Since energy is computed from the forces work, for the Newmark scheme, the oscillations mentioned in the introduction do not appear. Figures 7 and 8 illustrate the von Mises stress evolution for an element at the base of the beam. Results from the conservative scheme and the Newmark scheme are conform to the theory (i.e. constant von Mises stress). The accumulated number of iterations for one hundred time 


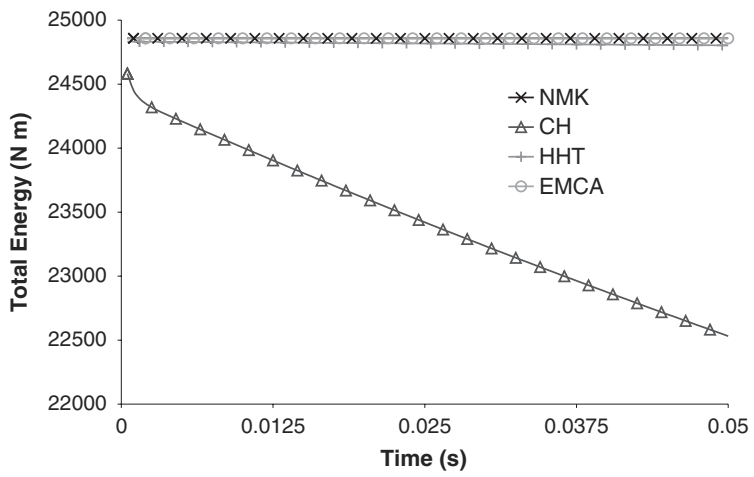

Figure 5. Total energy evolution for the beam in uniform rotation.

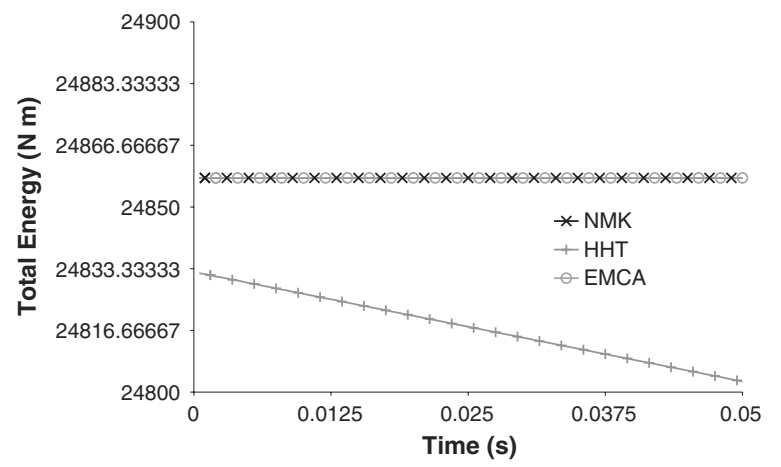

Figure 6. Total energy evolution for the beam in uniform rotation (zoom).

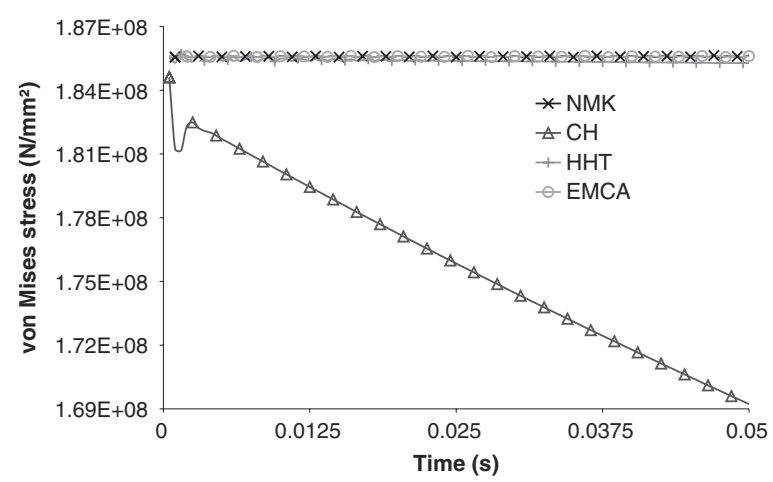

Figure 7. Evolution of the von Mises stress $\left(\mathrm{N} / \mathrm{mm}^{2}\right)$ at the base of the beam in uniform rotation.

steps are reported in Table II. The conservative scheme and the Newmark scheme converge in the same number of iterations. On the other hand, the Chung-Hulbert and the HilbertHughes-Taylor require more iterations since the numerical dissipation results in an angular acceleration of the beam. 


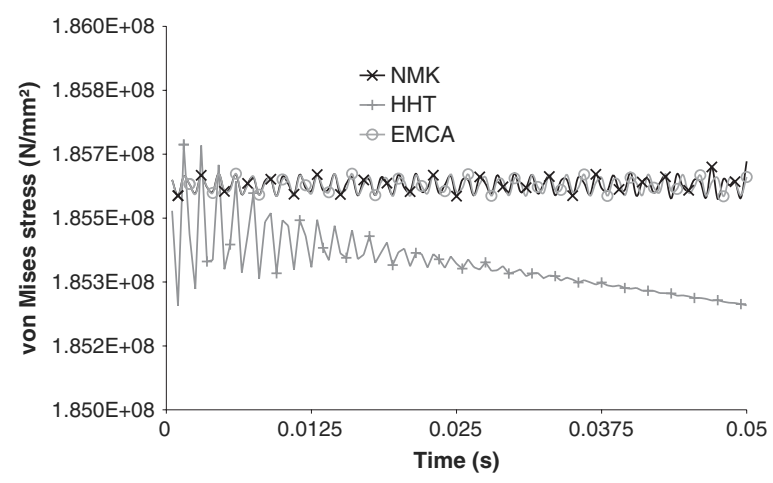

Figure 8. Evolution of the von Mises stress $\left(\mathrm{N} / \mathrm{mm}^{2}\right)$ at the base of the beam in uniform rotation (zoom).

Table II. Iterations number for the beam in uniform rotation (100 time steps).

\begin{tabular}{lc}
\hline Scheme & Iterations number \\
\hline Newmark & 302 \\
Chung-Hulbert & 400 \\
Hilbert-Hughes-Taylor & 424 \\
Conservative scheme & 300 \\
\hline
\end{tabular}

Table III. Properties of the Taylor bar problem.

\begin{tabular}{lc}
\hline Property & Value \\
\hline External diameter & $d_{e}=6.4 \mathrm{~mm}$ \\
Length & $l=32.4 \mathrm{~mm}$ \\
Density & $\rho=8930 \mathrm{~kg} / \mathrm{m}^{3}$ \\
Young's modulus & $E=117 E 9 \mathrm{~N} / \mathrm{m}^{2}$ \\
Poisson ratio & $v=0.35$ \\
Yield stress & $\sigma_{0}=400 \mathrm{~N} / \mathrm{mm}^{2}$ \\
Hardening parameter & $h=100 \mathrm{~N} / \mathrm{mm}^{2}$ \\
Initial velocity & $\dot{x}_{0}=227 \mathrm{~m} / \mathrm{s}$
\end{tabular}

\subsection{Example 2: The Taylor bar problem}

This classical example was first simulated with a conservative algorithm for a hyperelastic Saint-Venant-Kirchhoff material by Meng and Laursen [23]. It consists in a cylindrical bar (Table III), discretized by 576 elements (Figure 9). It has an initial velocity $\dot{x}_{0}$. The time step size is equal to $0.1 \mu$ s (small enough to avoid the obligation of taking into account the terms of the second order in the increment of the plastic deformation as previously mentioned. Figures 10 and 11 represent the evolution of the total energy (internal dissipation included). 


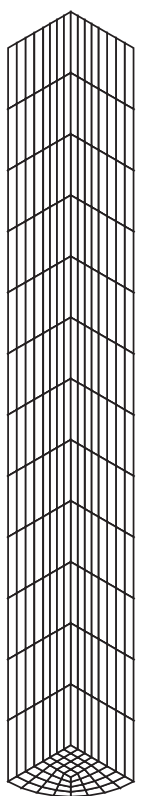

Figure 9. Discretization of the Taylor's bar.

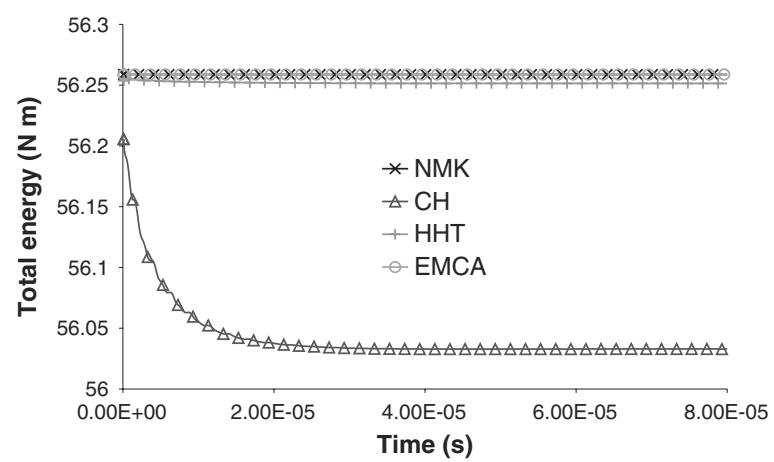

Figure 10. Evolution of the total energy for the Taylor bar problem.

Figures 12 and 13 represent the evolution of the internal dissipation. Let us note that the internal dissipation does not decrease during the time evolution. The second law of thermodynamics is therefore verified. It appears that the Chung-Hulbert and the Hilbert-HughesTaylor algorithms underestimate the internal dissipation. The final plastic strains are illustrated in Figure 14. The solution obtained by the Chung-Hulbert algorithm is $2 \%$ different of the other solutions. The differences between the schemes are rather small, resulting from the fact that the internal physical dissipation is much more important (275 times at the end of the computation for the Chung-Hulbert algorithm) than the numerical dissipation. Therefore the differences in the solutions obtained are not very important (less than $2 \%$ ). The number of 


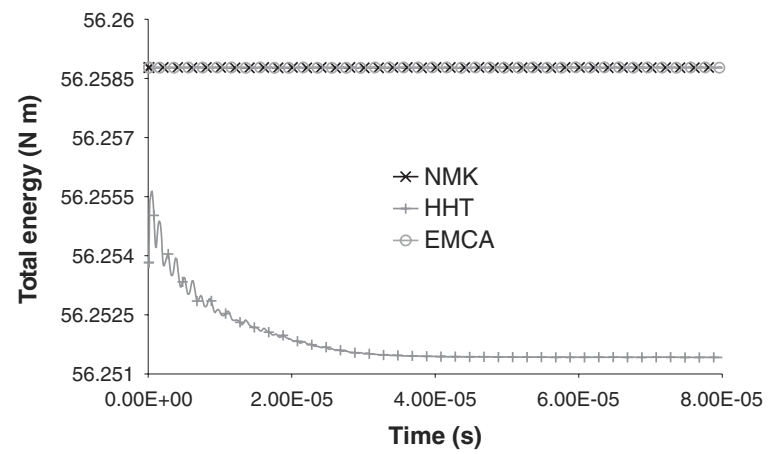

Figure 11. Evolution of the total energy for the Taylor bar problem (zoom).

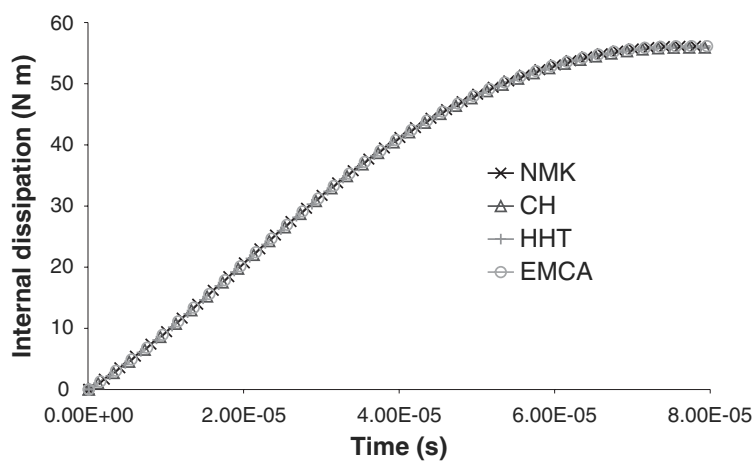

Figure 12. Evolution of the total internal dissipation for the Taylor bar problem.

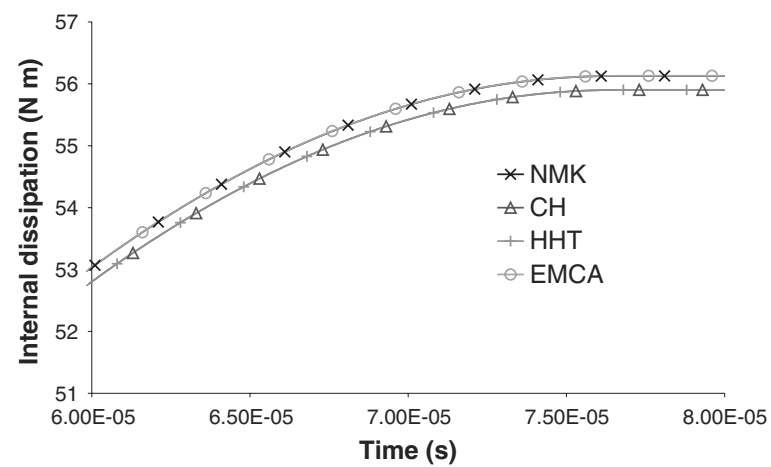

Figure 13. Evolution of the total internal dissipation for the Taylor bar problem (zoom).

iterations are reported in Table IV. The Newmark scheme is the most expensive one $(2.5 \%$ more than the conservative scheme, 5\% more than the Chung-Hulbert and 9\% more than the Hilbert-Hughes-Taylor scheme). The Chung-Hulbert algorithm is more expensive than the 
NMK

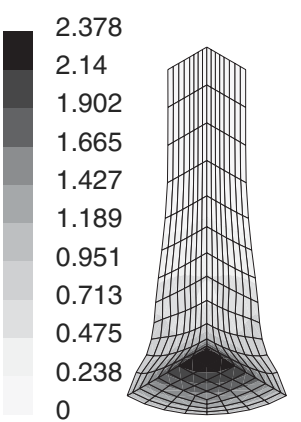

$\mathrm{HHT}$

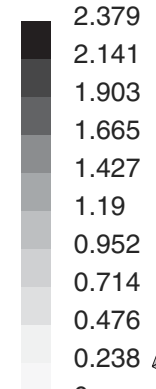

$\mathrm{CH}$

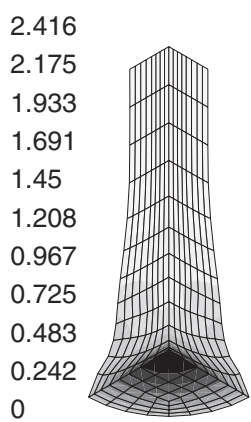

EMCA

Figure 14. Equivalent plastic strain for the Taylor bar after $80 \mu \mathrm{s}$.

Table IV. Iterations number of the Taylor's bar.

\begin{tabular}{lc}
\hline Scheme & Iterations number \\
\hline Newmark & 1951 \\
Chung-Hulbert & 1844 \\
Hilbert-Hughes-Taylor & 1777 \\
Conservative & 1904 \\
\hline
\end{tabular}

Hilbert-Hughes-Taylor algorithm, even if the numerical dissipation if higher. It comes from the severity of the tolerance on the residue $\left(10^{-10}\right)$ and the small time step size. Let us note that this difference in the number of iterations does not include the cost of the evaluation of the internal forces and of the stiffness matrix. Once the stress tensor has been evaluated, these evaluations are twice more expensive for the conservative scheme that for the other schemes. Results obtained are similar to the results obtained by Meng and Laursen [23].

\subsection{Example 3: The tumbling L-shaped block}

The dynamics of an elastoplastic L-shaped block is studied. This L-shaped block was discretized into 99 uniform elements. Its geometry is described in Figure 15. The properties 


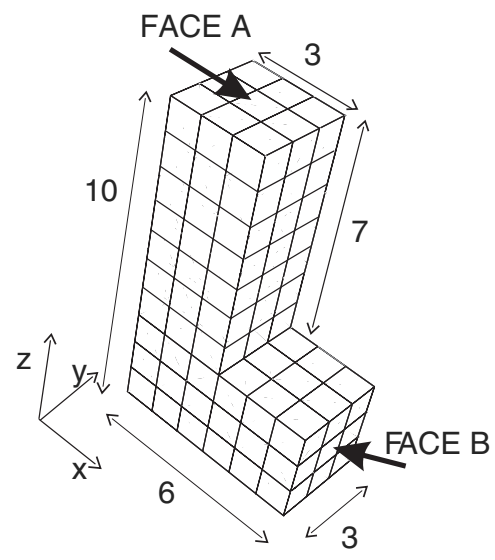

Figure 15. Geometry (m) of the tumbling L-shaped block.

Table V. Properties of the tumbling L-shaped block.

\begin{tabular}{lc}
\hline Property & Value \\
\hline Density & $\rho=100 \mathrm{~kg} / \mathrm{m}^{3}$ \\
Young's modulus & $E=2812 \mathrm{~N} / \mathrm{m}^{2}$ \\
Poisson ratio & $v=0.40625$ \\
Yield stress & $\sigma_{0}=300 \mathrm{~N} / \mathrm{m}^{2}$ \\
Hardening parameter & $h=400 \mathrm{~N} / \mathrm{m}^{2}$ \\
\hline
\end{tabular}

of the material are reported in Table V. On face A (see Figure 15), a force, depending on time $t$, is applied at each node. This time dependent force is given by

$$
\left(\begin{array}{c}
F_{x} \\
F_{y} \\
F_{z}
\end{array}\right)=\left(\begin{array}{c}
4 \\
8 \\
12
\end{array}\right) \mathrm{N} / \mathrm{s} \times\left\{\begin{array}{cc}
t, & 0 \leqslant t \leqslant 2.5 \mathrm{~s} \\
(5-t), & 2.5<t \leqslant 5 \mathrm{~s}
\end{array}\right\}
$$

On face B, another force is applied at each node

$$
\left(\begin{array}{c}
F_{x} \\
F_{y} \\
F_{z}
\end{array}\right)=\left(\begin{array}{c}
-4 \\
-8 \\
-12
\end{array}\right) \mathrm{N} / \mathrm{s} \times\left\{\begin{array}{cc}
t, & 0 \leqslant t \leqslant 2.5 \mathrm{~s} \\
(5-t), & 2.5<t \leqslant 5 \mathrm{~s}
\end{array}\right\}
$$

After $5 \mathrm{~s}$, the forces are relaxed. The time step size is equal to $0.25 \mathrm{~s}$.

Figure 16 represents the evolution of the total energy (internal dissipation included). Figure 17 represents the evolution of the internal dissipation. The numerical dissipation occurring for the Chung-Hulbert and the Hilbert-Hughes-Taylor algorithms leads to an underestimation of the internal dissipation. The final geometric configuration and the final plastic strain distribution are illustrated in Figure 18. Owing to the lack of accuracy resulting from the numerical 


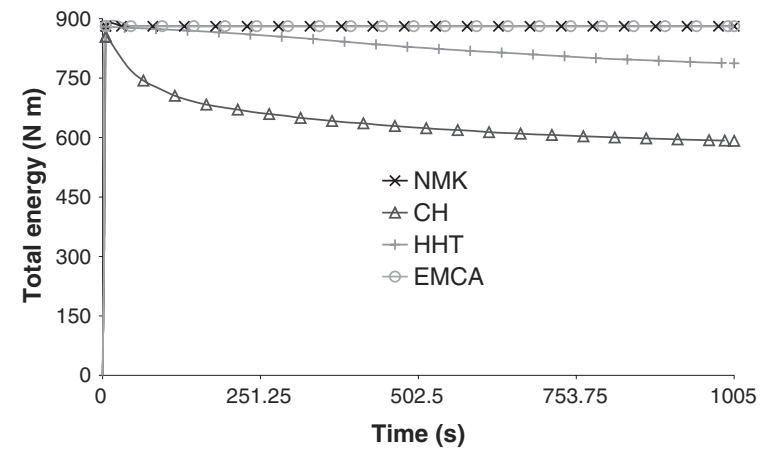

Figure 16. Evolution of the total energy for the tumbling L-shaped block.

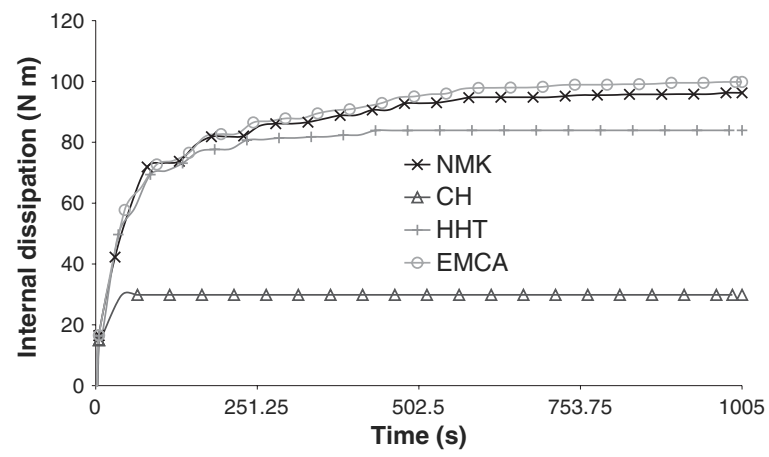

Figure 17. Evolution of the total internal dissipation for the tumbling L-shaped block.

dissipation, the Hilbert-Hughes-Taylor and the Chung-Hulbert solutions are different in the plastic strain but also in the final geometric configuration. The numbers of iterations are reported in Table VI. Each scheme leads to approximately the same cost with a difference of less than $0.05 \%$.

\section{CONCLUSIONS}

A new expression of the internal forces at the element level for hypoelastic materials was presented. When used with the conservative mid-point scheme, this expression leads to an energy-momentum conservative scheme. Moreover, the internal dissipation, resulting from the plastic deformation, is consistent with the laws of thermodynamic. If the problem remains elastic, our formulation is shown to be similar with the formulation proposed by Simo and Tarnow [12] for a Saint Venant-Kirchhoff hyperelastic material, but our formulation is more general in the sense that it remains valid for general hypoelastic-based $J 2$ plasticity models. Elastic and elastoplastic problems were presented. Note that, since the hypoelastic formulation provides a straightforward mechanism to extend small-strain constitutive models to non-linear kinematics, the proposed approach provides the great advantage of being applicable to virtually any other constitutive model (sophisticated hardening laws, damage, etc.). 

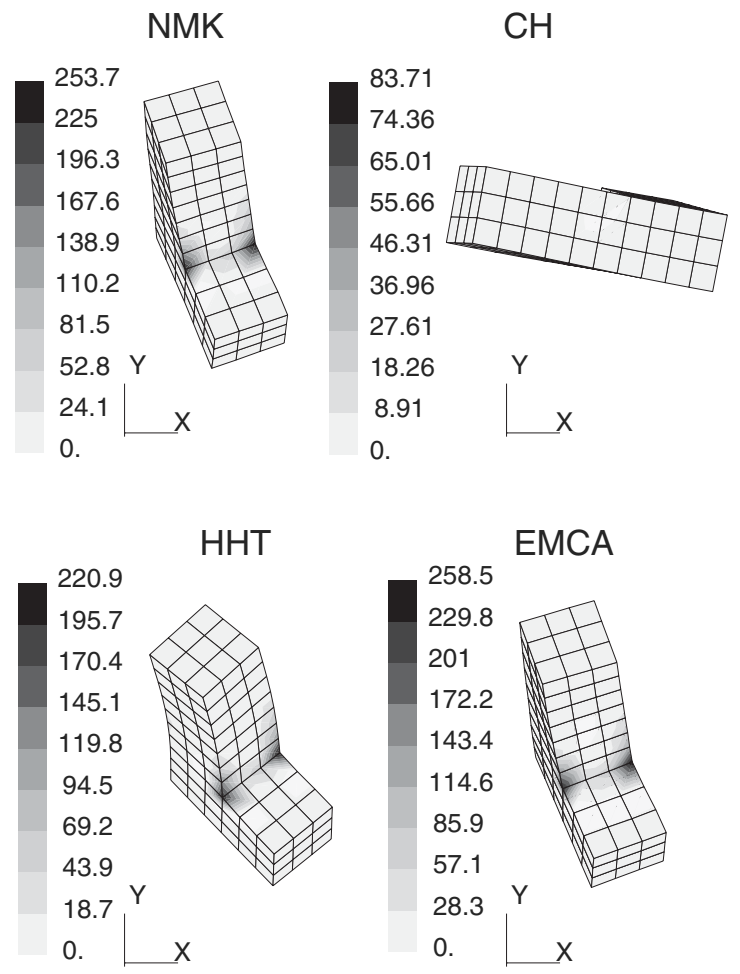

Figure 18. Equivalent plastic strain (magnified by $10^{3}$ ) for the tumbling L-shaped block after $1005 \mathrm{~s}$ (notice the different final configuration for $\mathrm{CH}$ and $\mathrm{HHT}$ ).

Table VI. Iterations number for the tumbling L-shaped block.

\begin{tabular}{lc}
\hline Scheme & Iterations number \\
\hline Newmark & 12093 \\
Chung-Hulbert & 12062 \\
Hilbert-Hughes-Taylor & 12053 \\
Conservative & 12087 \\
\hline
\end{tabular}

The solutions obtained with this conservative scheme were compared with the results obtained with the Newmark and the dissipative generalized- $\alpha$ algorithms. The conservative scheme ensured the conservation of the angular momentum, contrarily to the Newmark scheme that leads to numerical oscillations. When important plastic deformations (as in the Taylor bar problem) occur in a short time, the accuracy of the dissipative schemes are of the same order than the accuracy of the conservative scheme. For less-dissipative problems, the numerical dissipation leads to a loss of accuracy, principally due to the non-conservation of the 
angular momentum and could lead to an increase of the number of iterations. Moreover, this dissipative scheme can lead to instability in the non-linear range. Nevertheless, numerical dissipation can be useful when high frequency modes lead to failure of the conserving scheme. But this dissipation must be introduced in a controlled way (angular momentum conservation and positive numerical dissipation in the non-linear range).

If the iteration number of the conservative scheme is of the same order than for the other schemes (by about 10\%), the cost of the evaluation of the internal forces and of the stiffness matrix is higher (twice). But for large problems, this additional cost quickly becomes negligible when compared with the cost of matrix inversion.

In this paper, the second order terms in the expression of internal forces were not taken into account. In a further work, these terms will be integrated in the formulation. We will then be able to compare the difference between the Newmark algorithm and the proposed one for larger time step size.

\section{APPENDIX A: STIFFNESS MATRIX}

The stiffness matrix defined in (31) is evaluated. We assumed that applied forces $F_{\text {ext }}$ are conservative. First the expression $F_{\text {int }}^{*}$ is derived with respect to the positions at time $t_{n+1}$

$$
\begin{aligned}
\mathbf{K}^{* \xi \mu} & =\frac{\partial\left[F_{\text {int }}^{*}\right)^{\xi}}{\partial\left[x^{n+1}\right]^{\mu}} \\
& =\frac{1}{2} \int_{V_{0}}\left\{\frac{\partial \mathbf{F}_{n}^{n+1}}{\partial\left[x^{n+1}\right]^{\mu}} \Sigma^{n \mathrm{~T}} \mathbf{f}_{0}^{n \mathrm{~T}} D^{\xi} J_{0}^{n}\right\} \mathrm{d} V_{0}
\end{aligned}
$$

With relations (2) and (4), it leads to

$$
\frac{\partial \mathbf{F}_{n}^{n+1}}{\partial\left[x^{n+1}\right]^{\mu}} \mathbf{\Sigma}^{n \mathrm{~T}}=\mathscr{N} \cdot\left[B^{n}\right]^{\mu}
$$

with the first order tensor $\left[B^{n}\right]^{\mu}$ defined by $\left[B^{n}\right]^{\mu}=\mathbf{f}_{0}^{n \mathrm{~T}} D^{\mu}$, with the fourth order tensor $\mathscr{N}$ defined by $\mathscr{N}_{i j k l}=\mathbf{I}_{i k} \boldsymbol{\Sigma}_{j l}$, and with the operation $\left[B^{n}\right]^{\xi} \cdot \mathscr{N} \cdot\left[B^{n}\right]^{\mu}$ defined by

$$
\left[\left[B^{n}\right]^{\xi} \cdot \mathscr{N} \cdot\left[B^{n}\right]^{\mu}\right]_{i k}=\left[B^{n}\right]_{j}^{\xi} \mathscr{N}_{i j k l}\left[B^{n}\right]_{l}^{\mu}
$$

Therefore, using relation (A2), expression (A1) becomes

$$
\mathbf{K}^{* \xi \mu}=\frac{1}{2} \int_{V_{0}}\left\{\left[B^{n}\right]^{\xi} \cdot \mathscr{N} \cdot\left[B^{n}\right]^{\mu} J_{0}^{n}\right\} \mathrm{d} V_{0}
$$

Now the part $F_{\text {int }}^{* *}$ of the internal forces (46) is derived

$$
\begin{aligned}
\mathbf{K}^{* * \xi \mu} & =\frac{\partial\left[F_{\text {int }}^{* *}\right]^{\xi}}{\partial\left[x^{n+1}\right]^{\mu}}=\mathbf{K}^{1 \xi \mu}+\mathbf{K}^{2 \xi \mu} \\
\mathbf{K}^{1 \xi \mu} & =\frac{1}{2} \int_{V_{0}}\left\{\frac{\partial\left[\mathbf{I}+\mathbf{f}_{n}^{n+1}\right]}{\partial\left[x^{n+1}\right]^{\mu}} \boldsymbol{\Sigma}^{n+1} B^{\xi} J_{0}^{n+1}\right\} \mathrm{d} V_{0} \\
\mathbf{K}^{2 \xi \mu} & =\frac{1}{2} \int_{V_{0}}\left\{\left[\mathbf{I}+\mathbf{f}_{n}^{n+1}\right] \frac{\partial\left[\boldsymbol{\Sigma}^{n+1} B^{\xi} J_{0}^{n+1}\right]}{\partial\left[x^{n+1}\right]^{\mu}}\right\} \mathrm{d} V_{0}
\end{aligned}
$$


The first part of relation (A5) is evaluated. With the relation $\partial \mathbf{F}^{-1}=-\mathbf{F}^{-1} \partial \mathbf{F F} \mathbf{F}^{-1}$, it leads to

$$
\begin{aligned}
\frac{\partial \mathbf{f}_{n}^{n+1}}{\partial\left[x^{n+1}\right]^{\mu}} \mathbf{\Sigma}^{n+1} & =-\mathbf{F}_{0}^{n} \mathbf{f}_{0}^{n+1} \frac{\partial \mathbf{F}_{0}^{n+1}}{\partial\left[x^{n+1}\right]^{\mu}} \mathbf{f}_{0}^{n+1} \mathbf{\Sigma}^{n+1} \mathrm{~T} \\
& =-\mathscr{P} \cdot\left[B^{n+1}\right]^{\mu}
\end{aligned}
$$

with $\mathscr{P}_{i j k l}=\left[\mathbf{f}_{n}^{n+1}\right]_{i k} \boldsymbol{\Sigma}_{l j}^{n+1}$. Using (A6), the first part of (A5) is rewritten as

$$
\mathbf{K}^{1 \xi^{\xi \mu}}=-\frac{1}{2} \int_{V_{0}}\left\{\left[B^{n+1}\right]^{\xi} \cdot \mathscr{P} \cdot\left[B^{n+1}\right]^{\mu} J_{0}^{n+1}\right\} \mathrm{d} V_{0}
$$

The second part $\left(\mathbf{K}^{2 \xi \mu}\right)$ of relation (A5) is directly obtained from the stiffness matrix of the classical expression of the internal forces evaluated at configuration $n+1$. It leads [11] to

$$
\mathbf{K}^{2 \xi \mu}=\frac{1}{2} \int_{V_{0}}\left\{\left[\mathbf{I}+\mathbf{f}_{n}^{n+1}\right]\left[B^{(n+1)}\right]^{\xi} \cdot \mathscr{M}^{*} \cdot\left[B^{(n+1)}\right]^{\mu} J_{0}^{n+1}\right\} \mathrm{d} V_{0}
$$

with [11]

$$
\mathscr{M}_{i j k l}^{*}=\overline{\mathscr{M}}_{i j k l}+\boldsymbol{\Sigma}_{i j}^{n+1} \mathbf{I}_{k l}+\frac{1}{2}\left[\boldsymbol{\Sigma}_{l j}^{n+1} \mathbf{I}_{i k}-\boldsymbol{\Sigma}_{i k}^{n+1} \mathbf{I}_{j l}-\boldsymbol{\Sigma}_{i l}^{n+1} \mathbf{I}_{j l}-\boldsymbol{\Sigma}_{j k}^{n+1} \mathbf{I}_{i l}\right]
$$

where $\overline{\mathscr{M}}_{i j k l}$ is the material tensor characteristic of the material. In the elastic configuration, it is similar to the Hooke tensor. Otherwise, with $\mathbf{N}^{r}=\mathbf{R}_{n}^{n+1} \mathbf{N R}_{n}^{n+1}{ }^{\mathrm{T}}$, it becomes [44]

$$
\overline{\mathscr{M}}_{i j k l}=k \mathbf{I}_{i j} \mathbf{I}_{k l}+g^{*}\left[\mathbf{I}_{i l} \mathbf{I}_{j k}+\mathbf{I}_{i k} \mathbf{I}_{j l}-\frac{2}{3} \mathbf{I}_{i j} \mathbf{I}_{k l}-2 \mu^{*} \mathbf{N}_{i j}^{r} \mathbf{N}_{k l}^{r}\right]
$$

with $g^{*}=\beta g$ and

$$
\begin{aligned}
\beta & =\sqrt{\frac{2}{3}} \frac{\Sigma^{v, n+1}+\bar{\alpha}^{n+1}-\bar{\alpha}^{n}}{\sqrt{\left[\mathbf{s}^{\mathrm{e}}-\alpha\right]:\left[\mathbf{s}^{\mathrm{e}}-\alpha\right]}} \\
\mu^{*} & =\frac{g^{*}}{1+h /\left(3 g^{*}+[\beta-1] h\right)} \\
h & =\frac{\partial \Sigma^{v}}{\partial \varepsilon^{\mathrm{p}}}
\end{aligned}
$$

If $\mathscr{M}^{* *}$ is defined as

$$
\mathscr{M}_{i j k l}^{* *}=\left[\mathbf{I}+\mathbf{f}_{n}^{n+1}\right]_{\mathrm{im}} \mathscr{M}_{m j k l}^{*}
$$

Equation (A8) becomes

$$
\mathbf{K}^{2 \xi \mu}=\frac{1}{2} \int_{V_{0}}\left\{\left[B^{n+1}\right]^{\xi} \cdot \mathscr{M}^{* *} \cdot\left[B^{n+1}\right]^{\mu} J_{0}^{n+1}\right\} \mathrm{d} V_{0}
$$

The combination of terms (A4), (A7) and (A13) leads to the final expression of the stiffness matrix

$$
\mathbf{K}^{\xi \mu}=\frac{1}{4} \int_{V_{0}}\left\{\left[B^{n+1}\right]^{\xi} \cdot\left[\mathscr{M}^{* *}-\mathscr{P}\right] \cdot\left[B^{n+1}\right]^{\mu} J_{0}^{n+1}+\left[B^{n}\right]^{\xi} \cdot \mathscr{N} \cdot\left[B^{n}\right]^{\mu} J_{0}^{n}\right\} \mathrm{d} V_{0}
$$


Let us note that this stiffness matrix is not symmetric. Nevertheless, since relation (A9) (that is also used in the Newmark stiffness matrix [11]) gives a non-symmetric fourth order tensor, the use of the Newmark scheme also gives a non-symmetric stiffness matrix.

\section{ACKNOWLEDGEMENT}

L. Noels and L. Stainier wish to acknowledge support from the Belgian National Fund for Scientific Research (FNRS).

\section{REFERENCES}

1. Newmark N. A method of computation for structural dynamics. Journal of the Engineering Mechanics Division (ASCE) 1959; 85(EM3):67-94.

2. Belytschko T, Hughes T. Computational Methods for Transient Analysis. North Holland: Amsterdam, 1983.

3. Hughes T. The Finite Element Method. Prentice-Hall: Englewood Cliffs, NJ, 1987.

4. Belytschko T, Liu W, Moran B. Nonlinear Finite Elements for Continua and Structures. Wiley: New York, 2000.

5. Kane C, Mardsen JE, Ortiz M, West M. Variational integrators and the Newmark algorithm for conservative and dissipative mechanical system. International Journal for Numerical Methods in Engineering 2000; 49: 1295-1325.

6. Belytschko T, Schoeberle DF. On the unconditional stability of an implicit algorithm for non-linear structural dynamics. Journal of Applied Mechanics 1975; 42:865-869.

7. Hughes TJR. A note on the stability of Newmark's algorithm in nonlinear structural dynamics. International Journal for Numerical Methods in Engineering 1977; 11(2):383-386.

8. Simo JC, Tarnow N, Wong KK. Exact energy-momentum conserving algorithms and sympletic schemes for nonlinear dynamics. Computer Methods in Applied Mechanics and Engineering 1992; 100:63-116.

9. Chung J, Hulbert GJM. A time integration algorithms for structural dynamics with improved numerical dissipations: the generalized- $\alpha$ method. Journal of Applied Mechanics 1993; 60:371-375.

10. Géradin M, Cardona A. Flexible Multibody Dynamics: A Finite Element Approach. Wiley: New York, 2000.

11. Ponthot J-P. Traîtement unifié de la mécanique des milieux continus solides en grandes transformations par la méthode des éléments finis. Ph.D. Thesis, Université de Liège, 1995 (in French).

12. Simo JC, Tarnow N. The discrete energy-momentum method conserving algorithms for nonlinear elastodynamics. Zeitschrift für Angewandte Mathematik und Physik (ZAMP) 1992; 43:757-792.

13. Simo JC, Gonzalez O. Recent results on the numerical integration of infinite-dimensional hamiltonian system. In: Recent Developments in Finite Element Analysis, Hughes TJR, Onate E, Zienkiewicz OC (eds). CIMNE: Barcelona, Spain, 1994, pp. 255-271.

14. Simo JC, Tarnow N. A new energy and momentum conserving algorithms for the nonlinear dynamics of shells. International Journal for Numerical Methods in Engineering 1994; 37:2527-2549.

15. Zhong HG, Crisfield MA. An energy-conserving co-rotational procedure for the dynamics of shell structures. Engineering Computations 1998; 15(5):552-576.

16. Kuhl D, Crisfield MA. Energy-conserving and decaying algorithms in non-linear structural dynamics. International Journal for Numerical Methods in Engineering 1999; 45:569-599.

17. Sansour C, Wagner W, Wriggers P, Sansour J. An energy-momentum integration scheme and enhanced strain finite elements for non-linear dynamics of shells. International Journal of Nonlinear Mechanics 2002; 37: 951-966.

18. Brank B. An energy conserving non-linear dynamic finite formulation for flexible composite laminates. Computers and Structures 2002; 80:677-689.

19. Briseghella L, Majorana C, Pellegrino C. Conservation of angular momentum and energy in the integration of non-linear dynamics equations. Computer Methods in Applied Mechanics and Engineering 1999; 179:247-263.

20. Laursen T, Meng X. A new solution procedure for application of energy-conserving algorithms to general constitutive models in nonlinear elastodynamics. Computer Methods in Applied Mechanics and Engineering 2001; 190:6309-6322.

21. Gonzalez O, Simo JC. On the stability of sympletic and energy-momentum algorithms for non-linear hamiltonian systems with symmetry. Computer Methods in Applied Mechanics and Engineering 1996; 134:197-222.

22. Gonzalez O. Exact energy and momentum conserving algorithms for general models in nonlinear elasticity. Computer Methods in Applied Mechanics and Engineering 2000; 190:1763-1783.

23. Meng X, Laursen T. Energy consistent algorithms for dynamic finite deformation plasticity. Computer Methods in Applied Mechanics and Engineering 2001; 191:1639-1675. 
24. Armero F, Petocz E. Formulation and analysis of conserving algorithms for frictionless dynamic contact/impact problems. Computer Methods in Applied Mechanics and Engineering 1998; 158:269-300.

25. Armero F, Petocz E. A new dissipative time-stepping algorithm for frictional contact problems: formulation and analysis. Computer Methods in Applied Mechanics and Engineering 1999; 179:151-178.

26. Laursen TA, Chawla V. Design of energy conserving algorithms for frictionless dynamic contact problems. International Journal for Numerical Methods in Engineering 1997; 40:863-886.

27. Chawla V, Laursen T. Energy consistent algorithms for frictional contact problems. International Journal for Numerical Methods in Engineering 1998; 42:799-827.

28. Laursen T. Computational Contact and Impact Mechanics. Springer: Berlin, 2002.

29. Armero F, Romero I. Dissipative integration algorithms for nonlinear elastodynamics. In ECCM99, Proceedings of the European Conference on Computational Mechanics, Wunderlich W (ed.). Munich, Germany, 1999. CD-ROM.

30. Romero I, Armero F. High-frequency dissipative time-stepping algorithms for the dynamics of nonlinear shells. In: ECCOMAS 2000, Proceedings of the Congress on Computational Methods in Applied Sciences and Engineering, Oñate E, Brigeda G, Swález B. (eds). Barcelona, Spain, 2000.

31. Armero F, Romero I. On the formulation of high-frequency dissipative time-stepping algorithms for non-linear dynamics. part I: low-order methods for two model problems and nonlinear elastodynamics. Computer Methods in Applied Mechanics and Engineering 2001; 190:2603-2649.

32. Armero F, Romero I. On the formulation of high-frequency dissipative time-stepping algorithms for non-linear dynamics. part II: second-order methods. Computer Methods in Applied Mechanics and Engineering 2001; 190:6783-6824.

33. Ibrahimbegovic A, Mamouri S. Energy conserving/decaying implicit time-stepping scheme for non-linear dynamics of three-dimensional beams undergoing finite rotations. Computer Methods in Applied Mechanics and Engineering 2002; 191:4241-4258.

34. Hughes T, Caughey T, Liu W. Finite-element method for non-linear elastodynamics which conserve energy. Journal of Applied Mechanics 1978; 45:366-370.

35. Kuhl D, Ramm E. Constraint energy momentum algorithm and its application to non-linear dynamics of shells. Computer Methods in Applied Mechanics and Engineering 1996; 136:293-315.

36. Kuhl D, Ramm E. Generalized energy-momentum method for non-linear adaptive shell dynamics. Computer Methods in Applied Mechanics and Engineering 1999; 178:343-366.

37. Betsch P, Steinmann P. Inherent energy conserving time finite elements for classical mechanics. Journal of Computational Physics 2000; 160:88-116.

38. Betsch P, Steinmann P. Conservation properties of a time FE method. Part I: Time-stepping schemes for n-body problems. International Journal for Numerical Methods in Engineering 2000; 49:599-638.

39. Betsch P, Steinmann P. Conservation properties of a time FE method. Part II: Time-stepping schemes for non-linear elastodynamics. International Journal for Numerical Methods in Engineering 2001; 50:1931-1955.

40. Borri M, Bottasso L, Trainelli L. Integration of elastic multibody system by invariant conserving/dissipating algorithms. i. formulation. Computer Methods in Applied Mechanics and Engineering 2001; 190:3669-3699.

41. Bottasso L, Borri M, Trainelli L. Integration of elastic multibody system by invariant conserving/dissipating algorithms. ii. numerical schemes and applications. Computer Methods in Applied Mechanics and Engineering 2001; 190:3701-3733.

42. Nagtegaal J. On the implementation of inelastic constitutive equations with special reference to large deformation problems. Computer Methods in Applied Mechanics and Engineering 1982; 33:469-484.

43. Nagtegaal J, Veldpaus F. Analysis of metal forming problems with an improved finite strain plasticity formulation. Numerical Methods in Industrial Forming Processes. Pineridge: Swansea, UK, 1982.

44. Nagtegaal J, Veldpaus F. On the implementation of finite strain plasticity equations in a numerical model. In Numerical Analysis of Forming Processes, Pittman et al. (eds). Wiley: New York, 1984.

45. Ponthot J-P. Unified stress update algorithms for the numerical simulation of large deformation elasto-plastic and elasto-viscoplastic processes. International Journal of Plasticity 2002; 18:91-126.

46. Wilkins M. Calculation of elastoplastic flows. In: Methods of Computational Physics, Alder B (ed.). Academic Press: New York, 1964; 211-263.

47. Maenchen M, Sack S. The tensor code. In Methods of Computational Physics, vol. 3. Alder B (ed.). Academic Press: New York, 1964

48. Simo J, Hughes T. Computational Inelasticity. Springer: Berlin, 1998.

49. Lubliner J. Plasticity Theory. Macmillan: New York, 1990.

50. Hilbert H, Hughes T, Taylor R. Improved numerical dissipation for time integration algorithms in structural dynamics. Earthquake Engineering and Structural Dynamics 1977; 5:283-292. 